\title{
NUMERICAL MATRIX METHOD FOR SOLVING STATIONARY ONE-DIMENSIONAL SCHRÖDINGER EQUATION. AMSSE PROGRAM
}

\author{
WLODZIMIERZ SALEJDA, MARCIN JUST, MICHAL H. TYC \\ Institute of Physics, Wrockaw University of Technology \\ Wybrzeze Wyspiańskiego 27, 50-370 Wrockaw, Poland
}

\begin{abstract}
An implementation of numerical algebraic methods of solving a stationary one-dimensional Schrödinger equation (SODSE) is presented. In the framework of the proposed approach, SODSE is converted into an algebraic eigenvalue problem, which represents a discrete version of studied problem on an equally spaced grid. The AMSSE program written in Delphi calculates eigenvalues and corresponding eigenvectors by means of various methods and algorithms described here. It is an efficient and valuable computational environment, which can be used in science and nanotechnology. Arbitrary potentials can be introduced into AMSSE program in the form of analytic formulae or data tables, or with the mouse. The user-friendly graphical interface takes advantage of full capabilities of the Windows operating system. Main program features are described. Efficiency and accuracy of different numerical algorithms are comprehensively tested and compared. Factors influencing accuracy are discussed. Examples are widely presented. Matrix approach extension to the case of an effective-mass equation is mentioned.
\end{abstract}

\section{INTRODUCTION}

A fundamental problem of nonrelativistic quantum mechanics [1-9] is connected with solution of stationary one-dimensional Schrödinger equation (SODSE)

$$
-\frac{\hbar^{2}}{2 m} \frac{\mathrm{d}^{2} \Psi(x)}{\mathrm{d} x^{2}}+V(x) \Psi(x)=\varepsilon \Psi(x),
$$

where $V(x)$ is the potential energy and $m$ denotes the particle mass.

In general, SODSE cannot be solved analytically (exact solutions are known for special cases of potential energy $V(x)$, and discussed in standard quantum mechanics textbooks [1-9]). Therefore various numerical approaches have been proposed and applied [10-17], There exist different ways of solving SODSE numerically. These can be classified as follows:

(A) Finite element methods - equation (1) is solved on a finite set of subintervals and the obtained solutions are matched; the most popular and familiar method, called transfer matrix method [3, 13], belongs to this group.

(B) Variational Rayleigh-Ritz-Galerkin methods [1-3] - an approximation to the wave function $\Psi$ is represented as a basis function superposition (chosen in a reasonable way), and, from variational principle, the secular equation is derived and diagonalized [18-21], which gives approximations to eigenenergies of (1).

(C) Finite-difference methods, called also grid methods:

(CI) Shooting methods [12-13]; 
(C2) Matrix (or global) methods [10, 11, 15-17], which convert the Schrödinger equation (1) into an algebraic eigenproblem [18-21],

Both (C1) and (C2) methods are based on approximation of the second derivative of the wave function in (1) by finite-difference quotients [22],

The main difficulty in applying finite element (A), variational (B), or shooting (C1) methods is selection of a "good initial guess" for eigenvalues, eigenvectors or complete set of basis function. In this sense (A), (B) and (C1) methods are not universal. Therefore we prefer matrix approach (C2), which has the following advantages over the others ones: (1) no initial guess for eigenvalues or eigenvectors is needed to start computation; (2) neither iteration, matching nor relaxation procedure is employed; (3) one can use the most efficient and advanced computer algorithms worked out in numerical linear algebra [18-21].

In subsequent sections of this paper we describe:

(1) Finite difference approach allowing approximation of the second derivative in the Schrödinger equation (1) by central difference formulae [22];

(2) Matrix representations of SODSE on an equally spaced one-dimensional grid;

(3) Numerical algorithms implemented in our computer program called AMSSE [23] (Algebraic Methods of Solving Schrodinger Equation), written in Delphi;

(4) Main the AMSSE program capabilities;

(5) Graphical interface;

(6) Discussion of accuracy and efficiency of the algorithms implemented in the AMSSE program;

(7) Selected results of numerical calculations obtained by means of the AMSSE program;

(8) Matrix approach extension to the case of an effective-mass equation with arbitrary spatial electron effective-mass dependence.

The presented approach is an intuitive and very efficient technique useful for physicists and engineers working in solid state physics area, especially in physics of low-dimensional structures.

\section{DISCRETIZATION PROCEDURE AND MATRIX EIGENPROBLEM}

The discretization procedure is realized by introducing the grid of points

$$
x_{i}=a+i \cdot h, \quad i=0,1,2, \ldots, N+1,
$$

where $h=x_{i+1}-x_{i}=(b-a) /(N+1), N$ is a sufficiently large natural number, and $\langle a, b\rangle$ is the interval in which we solve SODSE. We choose the boundary conditions as follows: $\Psi($ a) $\sim \Psi(b)=0$ which, from the physical point of view, means that $V(a)=V(b)=\infty$ (i, e., we place infinite barriers at the ends of interval $\langle a, b\rangle$ ). These boundary conditions ensure that the matrix of the algebraic eigenproblem will have a band form.

Next, the central finite-difference formulas are applied in order to approximate the secondderivative in (1) at $x=x_{i}$ point which can be taken as [22] 


$$
\begin{gathered}
\Psi^{\prime \prime}\left(x_{i}\right)=\Psi_{i}^{\prime \prime}=\left.\frac{\mathrm{d}^{2} \Psi}{\mathrm{d} x^{2}}\right|_{x=x_{i}}=\frac{\Psi_{i+1}+\Psi_{i-1}-2 \Psi_{i}}{h^{2}}+O\left(h^{2}\right) \\
\Psi^{\prime \prime \prime}\left(x_{i}\right)=\Psi_{i}^{\prime \prime}=\left.\frac{\mathrm{d}^{2} \Psi^{2}}{\mathrm{~d} x^{2}}\right|_{x=x_{i}}=\frac{-\Psi_{i+2}+16 \Psi_{i+1}-30 \Psi_{i}+16 \Psi_{i-1}-\Psi_{i-2}}{12 h^{2}}+O\left(h^{4}\right) .
\end{gathered}
$$

Let us note that (3) and (4) are three- and five-point central finite-difference formulae, respectively.

Now, using formula (3) or (4), for each grid points (2) we can rewrite differential equation (1) as an algebraic eigenvalue problem

$$
M_{T} \vec{\Psi}=\tilde{\varepsilon} \cdot \vec{\Psi}
$$

where $M_{T}$ is the symmetric tridiagonal matrix which we write in an abbreviated form $M_{T}=$ $\operatorname{trid}\left(b_{i}, a_{i}, b_{i+1}\right)=\operatorname{trid}\left(-1,2+\tilde{V}\left(x_{i}\right),-1\right)$ (note that $\left(b_{i}, a_{i}, b_{i+1}\right)$ denotes the z-th row of the ti idiagonal matrix and the tilde over a symbol indicates corresponding renormalization), or

$$
M_{P} \ddot{\Psi}=12 \tilde{\varepsilon} \cdot \vec{\Psi}
$$

where $M_{P}$ is a symmetric pentadiagonal matrix which can be written also in abbreviated form; $M_{P}=\operatorname{pentad}\left(c_{i}, b_{i}, a_{i}, b_{i+1} c_{i+1}\right)=\operatorname{pentad}\left(1,-16,30+12 \widetilde{V}\left(x_{i}\right),-16,1\right) ;\left(\begin{array}{llll}c_{i}, & b_{i}, a_{i}, b_{i+1},\end{array}\right.$ $\left.c_{i+2}\right)$ is the $i$-th row of the pentadiagonal matrix, $\tilde{\varepsilon}=2 m \varepsilon h^{2} / \hbar^{2}$ and $\tilde{V}\left(x_{i}\right)=2 m V\left(x_{i}\right) h^{2} / \hbar^{2}$. We point out that $\vec{\Psi}^{\prime}$ denotes a one-column matrix, i. e.,

$$
\vec{\Psi}=\left(\Psi\left(x_{1}\right), \Psi\left(x_{2}\right), \Psi\left(x_{3}\right), \ldots, \Psi\left(x_{N}\right)^{T}=\left(\Psi_{1}, \Psi_{2}, \Psi_{3}, \ldots, \Psi_{N}\right)^{T}\right.
$$

(the upper index $T$ denotes the transposition).

The most general approach to the SODSE solution problem in the finite-difference schemes has been proposed by Gaurdiola and Ros [15], In this method the second derivative operator is represented by the Padé approximant $[n / m]\left(\hat{\delta}^{2}\right)$ [24], being an operator function of the tridiagonal matrix $\hat{\delta}^{2}=\operatorname{trid}(1,-2,1)$, the eigenvalues and eigenvectors of which are known exactly [15]. In the framework of this approach the Schrödinger equation (1) is converted into an algebraic eigenproblem

$$
A \vec{\Phi}=\left(D+h^{2} \vec{V}\right) \Phi=\tilde{\varepsilon} \vec{\Psi},
$$

where. $A=D+h^{2} \bar{V}$ is the dense symmetric matrix, $D$ is the diagonal matrix

$$
D_{i}=\frac{B_{i}}{C_{i}}, \quad B_{i}=\sum_{j=1}^{n}(-4)^{j} \lambda_{j} \sin ^{2 j}\left(\frac{i \pi}{2(N+1)}\right), \quad C_{i}=\sum_{j=1}^{m}(-4)^{j} \lambda_{j} \sin ^{2 j}\left(\frac{i \pi}{2(N+1)}\right)
$$

and elements of matrix $\vec{V}$ are given by 


$$
\bar{V}_{i j}=\frac{.2}{N+1} \sum_{l=1}^{N} \sin \left(\frac{i \cdot l \cdot \pi}{N+1}\right) \tilde{V}_{l} \sin \left(\frac{j \cdot l \cdot \pi}{N+1}\right)
$$

and

$$
\lambda_{j}=-4 \sin ^{2}\left(\frac{j \cdot \pi}{2(N+1)}\right)
$$

denotes the $j$-th eigenvalue of the tridiagonal symmetric $N$ × $N$ matrix $\hat{\delta}^{2}=\operatorname{trid}(1,-2,1)$.

This way we have transformed the original differential equation (1) into an algebraic eigenvalue problem defined by (5), (6) or (7). Values of $\varepsilon$, for which nonzero solutions of quations given by (5), (6) or (7) exist, are called eigenvalues, and the corresponding solutions are the eigenvectors. So, energy levels and wave functions occurring in SODSE is reduced to a computation of eigenvalues and eigenvectors of tridiagonal (5), pentadiagonal (6) or dense (7) symmetric matrices.

\section{NUMERICAL ALGORITHMS IMPLEMENTED IN AMSSE}

We enumerate and describe briefly the routines and algorithms implemented in the AMSSE program [23] for computation of eigenvalues and eigenvectors of algebraic eigenproblems (5), (6) or (7).

1. Standard algorithm (SA) - this subprogram uses EISPACK routines TQL2 or TQLRAT [18-21] (based on QR or QL decomposition); note that SA calculates all eigenvalues and eigenvectors of the tridiagonal matrix (5).

2. Sturm-Martin-Dean algorithm (SMDA) [11, 25, 26] - selected (by the user) eigenvalues of (5) are calculated by means of the Sturm-Martin-Dean sequences, applying a bisection procedure. The approach is equivalent to the negative-eigenvalue theorem called also the Sylvester theorem [26], A very efficient new algorithm based on binary search has been applied.

3. EISPACK routine (ER) - the subprogram uses EISPACK routine [21], which calculates selected number of eigenvalues $M_{T}$ in (5) by the bisection method.

4. Modified Sturm-Padé algorithm (MSPA) [23] - a chacteristic polynomial defined by the Sturm sequence is used in order to calculate selected (by the user) eigenvalues of (5). This is also a new algorithm, which uses quick binary search [23] for determination of the interval where characteristic polynomial changes its sign. Instead of applying the bisection method (applied in SMDA), we approximate the characteristic polynomial by its Padé approximant [1/1](x) [24] on the interval $\left\langle\alpha_{i}, \beta_{i}\right\rangle$ and then we find eigenvalue as a zero of the approximant.

5. Improved Sturm-Martin-Dean algorithm (ISMDA) [23] - selected (by the user) eigenvalues of pentadiagonal matrix (6) are calculated by means of the Sturm-Martin-Dean sequences and negative-eigenvalue Martin-Dean theorem [25], 
6. Lindberg algorithm (LA) [16] - the second derivative in SODSE is approximated by the Pade approximant

$$
[1 / 1]\left(\hat{\delta}^{2}\right)=\frac{1}{h^{2}} \cdot \frac{\hat{\delta}^{2}}{1+\hat{\delta}^{2} / 12},
$$

which allows us to convert (7) into a matrix eigenproblem

$$
A^{(q-s)} \vec{\Phi}=\tilde{\varepsilon} \vec{\Phi}
$$

with a quasi-symmetric tridiagonal matrix [19] $A^{(q-s)}$ the obtained eigenproblem (8) is further analysed by means of the Sturm sequences (described above); eigenvalues are found by the bisection method.

7. Modified Lindberg algorithm (MLA1) [23] - a characteristic polynomial and its derivative given by the Sturm sequences are used for derivation of selected eigenvalues of algebraic eigenproblem (7); the eigenvalues are found by the Newton-Raphson method.

8. Modified Lindberg algorithm (MLA2) [23] - a characteristic polynomial and its derivative given by the Sturm sequences are used for derivation of selected eigenvalues of algebraic eigenproblem (7); the eigenvalues are found by the secant method.

9. Improved Lindberg algorithm (ILA) [23] - a characteristic polynomial defined by the Sturm sequence is used in order to calculate selected eigenvalues of (7). This new algorithm is worked out by us and uses also quick binary search for detenu;nation of the interval where characteristic polynomial changes its sign. Instead of applying the bisection method, we approximate the characteristic polynomial by its Padé approximant [1/1] (x) [24] on the interval $\left\langle\alpha_{i}, \beta_{i}\right\rangle$, and then find an eigenvalue as a zero of Padé approximant. We have verified numerically that this method is accurate, very fast and the most efficient among implemented algorithms.

10. Guardiola-Ros algorithm (GRA) [15, 23] - for a given potential energy an algebraic eigenproblem (7) is solved using the Padé approximant

$$
[3 / 2]\left(\hat{\delta}^{2}\right)=\frac{1}{h^{2}} \cdot \frac{\hat{\delta}^{2}\left(1+49 \hat{\delta}^{2} / 276+79 \hat{\delta}^{4} / 28980\right)}{1+6 \hat{\delta}^{2} / 23+43 \hat{\delta}^{4} / 3220},
$$

as approximation of second derivative in (1). The obtained dense algebraic eigenproblem is solved by means of standard numerical routines [18-21],

Now let us present briefly an algorithm for calculation of eigenvectors corresponding to a given eigenvalue. In our AMSSE program we have implemented algorithm proposed by Dy, $\mathrm{Wu}$, Spratlin and Zheng in [27, 28] (see also [11, 23, 25]) and called further in the paper DWSZ algorithm, which allows to calculate eigenvector in the following steps:

EV1. The $i$-th eigenvalue $\varepsilon_{i}$ is computed by means of the algorithms (1-5) described above. EV2. Two sequences of numbers are calculated: 


$$
\Delta_{1}^{(-)}(i)=\frac{1}{a_{1}-\varepsilon_{i}}, \quad \Delta_{l}^{(-)}(i)=\frac{1}{a_{l}-\varepsilon_{i}-b_{l}^{2} \Delta_{l-1}^{(-)}}, \quad l=2,3,4, \ldots, N-1
$$

and

$$
\Delta_{N}^{(+)}(i)=\frac{1}{a_{N}-\varepsilon_{i}}, \quad \Delta_{l}^{(+)}(i)=\frac{1}{a_{l}-\varepsilon_{i}-b_{l+1}^{2} \cdot \Delta_{l+1}^{(+)}}, \quad l=N-1, N-2, N-3, \ldots, 2
$$

EV3. We place at pivoting coordinate $x_{m}$

$$
\Psi^{(i)}\left(x_{m}\right)=\Psi_{m}^{(i)}=1
$$

and we calculate the rest of coordinates of the $i$-th eigenvector using formulae

$$
\begin{gathered}
\Psi_{k}^{(i)}=-b_{k} \Delta_{k}^{(+)} \Psi_{k-1}^{(j)}, \quad k=m+1, \quad m+2, \quad m+3, \ldots, N \\
\Psi_{k}^{(i)}=-b_{k+1} \Delta_{k}^{(-)} \cdot \Psi_{k+1}^{(i)}, \quad k=m-1, \quad m-2, \quad m-3, \ldots, 1
\end{gathered}
$$

EV4. The magnitude of expression

$$
\mu_{m}^{(i)}=b_{m} \cdot \Psi_{m-1}^{(i)}+\left(a_{m}-\varepsilon_{i}\right) \Psi_{m}^{(i)}+b_{m+1} \Psi_{m+1}^{(i)}
$$

is calculated.

Now the main problem is connected with correct index $m$ for which $\mu_{m}^{(i)}$ is of order of a machine epsilon. In order to find an optimum index, the AMSSE program searches for the index $m^{\prime}$ for which magnitude $\left|\mu_{m^{\prime}}{ }^{(i)}\right|$ is minimal. We have verified numerically, that this way of calculations is very efficient and stable. Taken $m=m^{\prime}$, components of eigenvector are computed according to (9d-f).

\section{COMPUTING CAPABILITIES OF AMSSE PROGRAM}

The main purpose of our efforts was to create an universal, very efficient tool working under control of Windows 9x/NT/2000 systems for solving one-dimensional stationaiy Schrödinger equation, simple in use due to clear graphic user interface. We placed a special emphasis on different methods of inserting the potential energy into the computing environment 
(with formulae, graphically - with the help of mouse, or by means of data tables) as well as on comfort, efficiency and high computation precision.

The AMSSE program has been created using Delphi. It is universal tool giving a user possibility of solving stationaiy Schrödinger equation with any type and shape of one-dimensional potential wells. The AMSSE program has numerous advantages making it very efficient scientific and didactic instrument in the courses on quantum mechanics and physics of low-dimensional systems. The advantages making this program outstanding are:

- Graphical interface - the AMSSE program works under control of Windows 9x/NT/2000 operating systems. An intuitive arranging of control elements makes all operations easy and enables of a quick modification of all parameters (even to beginning user). Permanent visual control of a computations process and immediate stopping it at any time without any loss give a user a satisfaction of full control over the program. Graphical presentation of results on results on a display screen makes working with the program comfortable and easy.

- Full integration with an operating system - the AMSSE program realizes OLE server function, which makes it possible to access to its computational functions from other Windows programs. Multitasking and clipboard services make data exchange with other applications fast and easy.

- Co-operation with Microsoft Office gives a simple way of using the computational results in user's own publications.

- Numerous set of solving procedures and methods allow us to find eigenvalues with user's specified precision, and to compare results obtained by different algorithms and methods.

- Unusual efficiency of computations achieved by far-reaching algorithms.

- Flexibility in methods of defining the potential shape. The potential energy can be defined:

- by formula;

- as a function graph drawn on the screen with mouse;

- by imported from MS Excel.

- Built-in compare functions allow us to test and verify an influence of the potential shape or magnitudes of parameters on eigenvalues and eigenvectors.

- Capability of stopping computations in any moment and saving the results allows us to continue the work next time. It is possible to save the whole worksheet or only eigenvalues, potential or eigenvector.

- Quick and easy parameter modification, that concers:

- potential energy,

- integration range,

- computation precision.

- Simple and natural way to supply units and whole formulae. User can apply popular eV (electronvolts) and nanometers. A few basic physical constants are available and formulae could be entered in pure book-like form. 
The features specified above make the AMSSE program very useful tool for scientists and nanotechnologists, as well as for stutends.

\section{AMSSE PROGRAM GRAPHICAL INTERFACE}

In this section we describe briefly main components and principle of the AMSSE program. Figure 1 presents a short description of the main window of the program.

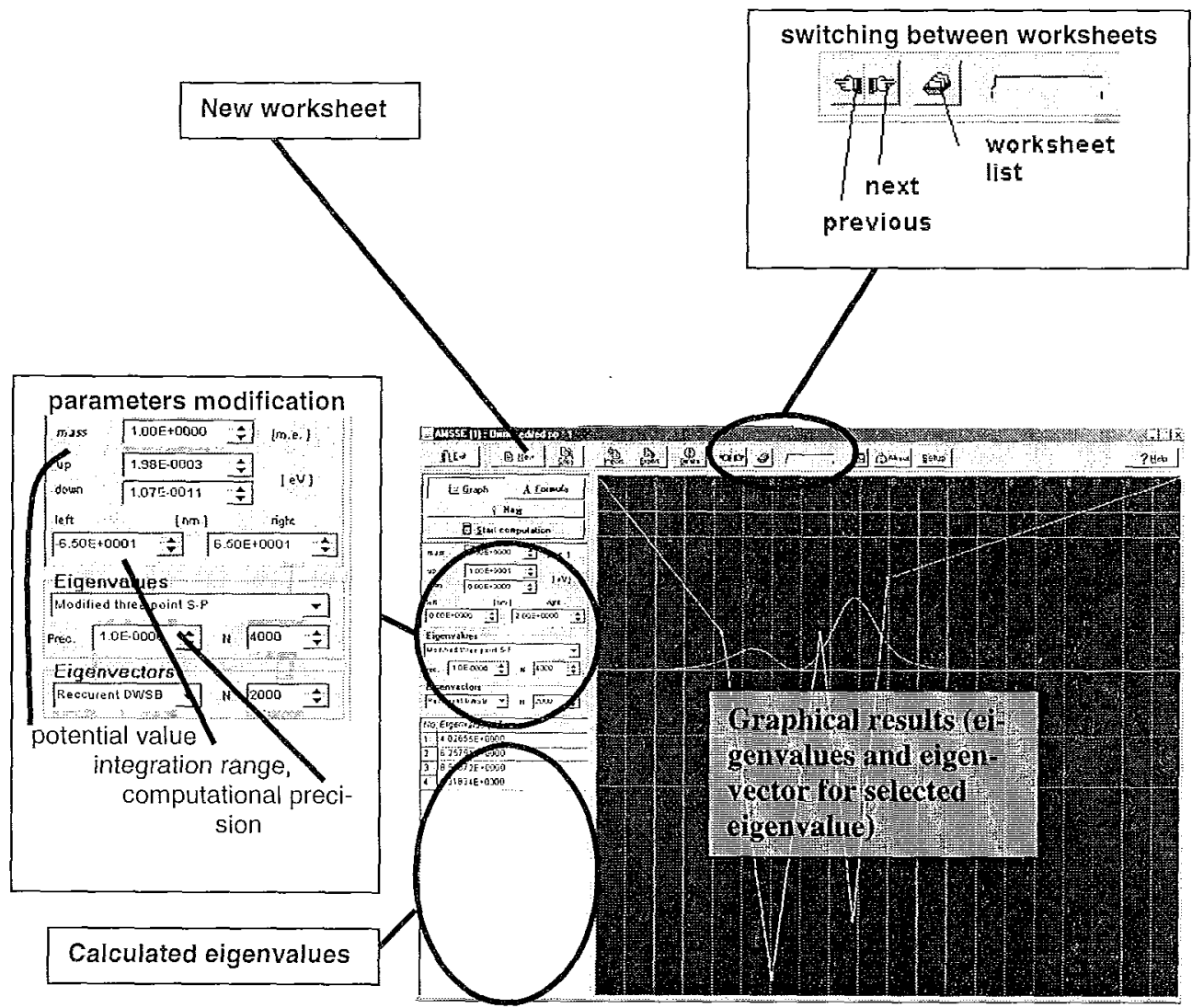

Fig. 1. Main window of the AMSSE's graphical interface

To start computations, one needs to perform a few steps shown below in Fig. 2.

The numerical solution procedure of SODSE (1) with the help of the AMSSE program consists of a few steps: (I) The potential energy is entered; (II) Magnitudes of parameters are defined; (III) Selected eigenvalues are calculated by selected algorithm; (IV) Normalized eigenvector corresponding to indicated eigenvalue is computed and displayed. 


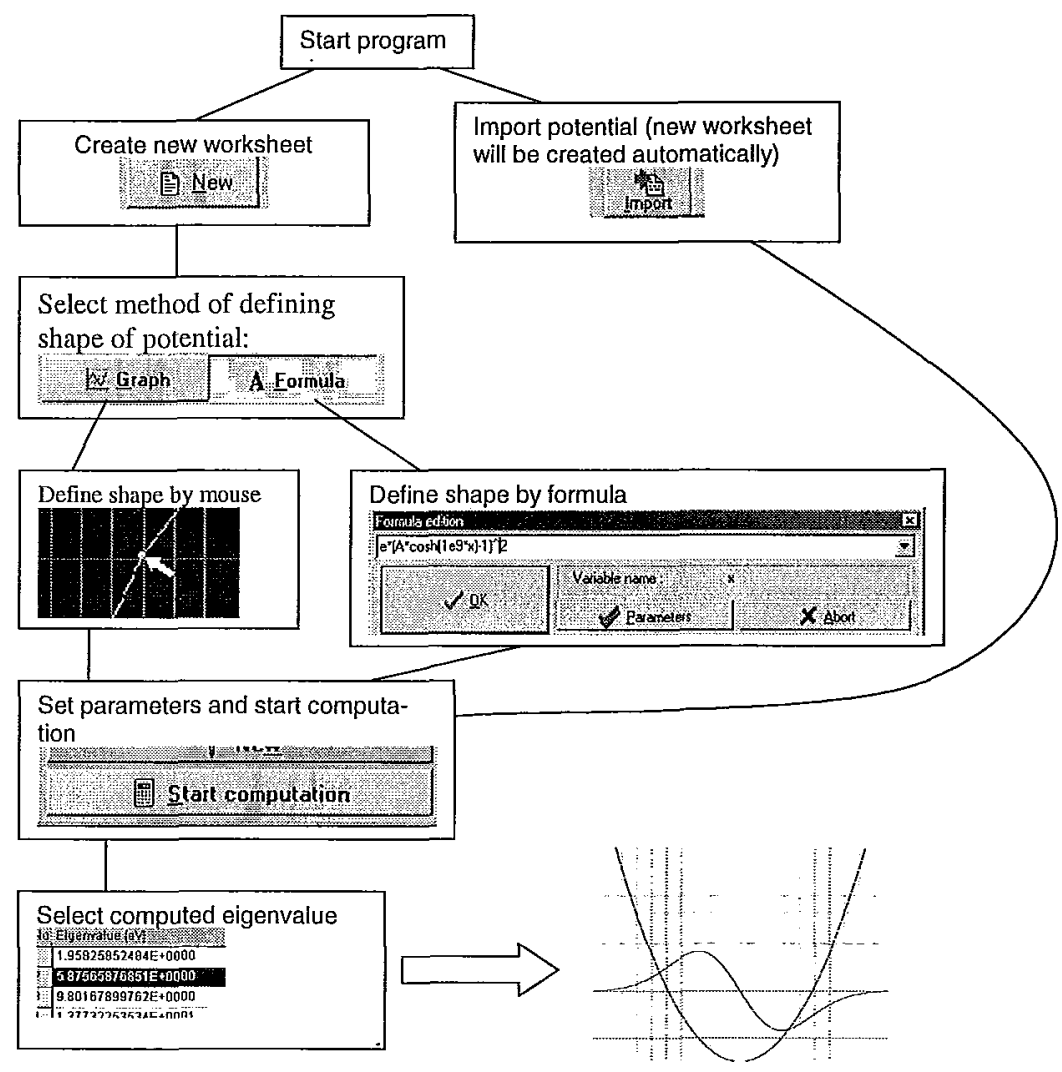

Fig. 2. Computational steps, which a user of the AMSSE program has to execute in order to solve SODSE

\section{TESTING OF THE NUMERICAL PROCEDURES}

The AMSSE program is a universal program which has neither possibility of measuring computation time, nor tools for comparing its output with analytical results. Despite this, extensive tests with the help of advanced tools has been performed in order to determine optimum application range of each implemented method for various classes of potentials. Numerical results were compared with analytical ones for selected analytically solvable problems.

Because a large number of parameters for the tested methods and potentials, the testing procedure had to be automated. One of the testing tools is PTEST1 program, which calls procedures from the AMSSE program and compares accuracy and efficiency of the algorithms for a given number of grid points $N$. It estimates the optimum $N$ for each algorithm as well. PTEST1 performs tests for:

1. the parabolic potential (quantum harmonic oscillator),

2. the radial equation for the Coulomb potential (hydrogen atom),

3. the Morse potential,

4. the Konwent potential (symmetric double-well). 
Test results are displayed on the screen (an example is shown in Table I).

Wider range of tests can be performed using Visual Basic macros contained in a special worksheet written for Microsoft Excel. Their results are displayed in the form of graphs and tables without any user's action.

For the testing purposes, we have defined the efficiency coefficient (EC) as

$$
\mathrm{EC}=\frac{1}{\sqrt{\text { achieved_accuracy }} \cdot(\text { execution_time_in_ms) }}
$$

The tests consist of two steps, which allows us to estimate a true performance of each method:

1. Determination of the maximum accuracy for a given number of grid points $N$ (by performing the calculations with full hardware precision - about 15 significant digits) and comparison the three lowest eigenenergies with analytical results,

2. Measurement of the computation time for the accuracy determined in the previous step.

Table I. Example results from the PTEST 1 application for the harmonic oscillator potential

\begin{tabular}{lrrrr}
\hline $\begin{array}{c}\text { Method's } \\
\text { name }\end{array}$ & $\begin{array}{c}\text { Execution time } \\
{[\mathrm{ms}]}\end{array}$ & Accuracy & Efficiency & \multicolumn{1}{c}{$N$} \\
\hline MSPA & 66 & $2.079 \mathrm{E}-06$ & 10.5 & 4000 \\
SMDA & 121 & $9.150 \mathrm{E}-06$ & 2.7 & 2000 \\
LA & 82 & $2.185 \mathrm{E}-08$ & 82 & 500 \\
MLA1 & 193 & $4.171 \mathrm{E}-12$ & 2537 & 4000 \\
MLA2 & 187 & $4.057 \mathrm{E}-12$ & 2655 & 4000 \\
ILA & 143 & $4.056 \mathrm{E}-12$ & 3473 & 4000 \\
S.A. & 428 & $3.322 \mathrm{E}-05$ & 0.41 & 1000 \\
ER & 98 & $5.485 \mathrm{E}-05$ & 1.4 & 2000 \\
ISMDA & 110 & $4.787 \mathrm{E}-08$ & 41 & 500 \\
GRA & 99 & $3.070 \mathrm{E}-12$ & 5765 & 100 \\
\hline
\end{tabular}

In the case of the harmonic oscillator potential, the Guardiola-Ros algorithm appears to be the most efficient one.

The form of the potential energy function is essential for the achieved accuracy. Five different potentials, representing typical cases and giving wide spectrum of behaviour of the algorithms, have been selected for testing. All of them are binding potentials (with local minima) and have one-dimensional form. They are briefly described below.

\subsection{Quantum harmonic oscillator}

Eigenenergies in a parabolic well are equally spaced (cf., Fig. 3), according to the formula 


$$
E_{n}=\hbar \omega \cdot\left(n+\frac{1}{2}\right)
$$

and therefore it is an ideal case for testing.

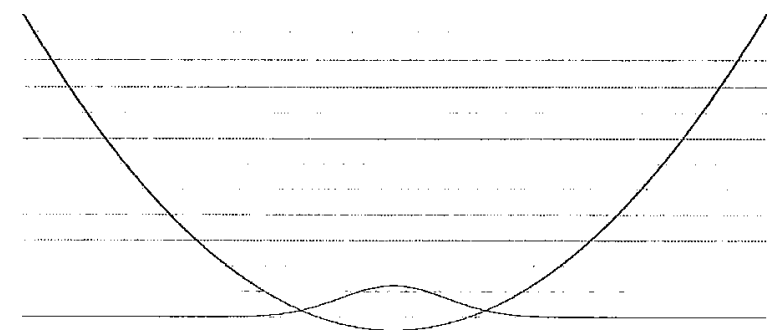

Fig. 3. The graph of the oscillator potential with eigenenergies and the ground state wave function, generated by the AMSSE program

\section{2. Three-dimensional Coulomb potential}

It is a potential of $1 / r$ type, which can be reduced to one-dimensional form [1] due to its spherical symmetry. This problem corresponds to a hydrogen atom and the Wannier-Mott exciton (in solid state physics). Its one-dimensional form reads

$$
V(x)=\frac{\hbar^{2} l(l+1)}{2 m_{e} x^{2}}-\frac{e^{2}}{4 \pi \varepsilon_{0} x},
$$

where $e$ is the elementary charge, $m_{e}$ is the electron mass and $l$ is the orbital angular momentum quantum number. The first term in the potential energy is connected with particle's angular momentum. The eigenvalues are

$$
E_{n}=-\frac{1}{n^{2}} \frac{m_{e}}{32} \frac{e^{4}}{\pi^{2} \varepsilon_{0}^{2} \hbar^{2}} .
$$

Two cases have been tested separately:

- $l=0-$ a potential with a $-1 / x$ singularity at $x=0$, which makes the computation difficult

(Fig. 4a)

- $l=2-$ potential with a minimum and a singularity of $+1 / x^{2}$ type (Fig. $4 \mathrm{~b}$ )

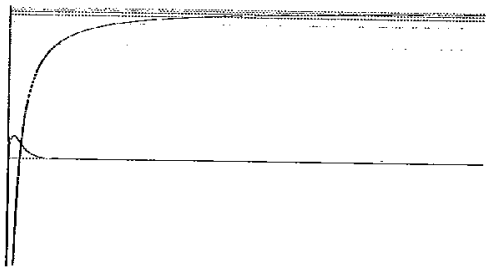

(a)

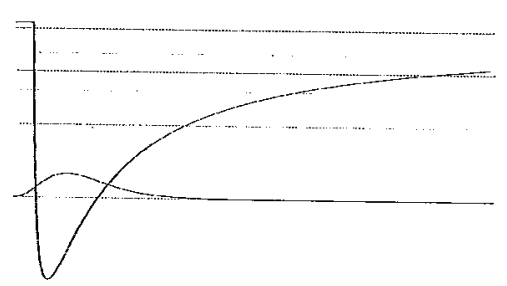

(b)

Fig. 4. The graph of the Coulomb potential [(a) $l=0$; (b) $l=2]$ with eigenenergies and the wave functions of the lowest states, generated by the AMSSE program 


\section{3. Morse potential}

This potential has been selected due to its application in physical chemistry [1], It has the form

$$
V=V_{0} \cdot\left(1-e^{-\alpha \cdot x}\right)^{2}
$$

and its eigenvalues are

$$
E_{n}=A \cdot\left((2 n+1)-\frac{A \cdot(2 n+1)^{2}}{4}\right), \quad \text { where } A=\frac{\alpha \hbar}{\sqrt{2 m V_{0}}} .
$$

The Morse potential, its eigenenergies and the ground state wave function computed by the program are shown in Fig. 5.

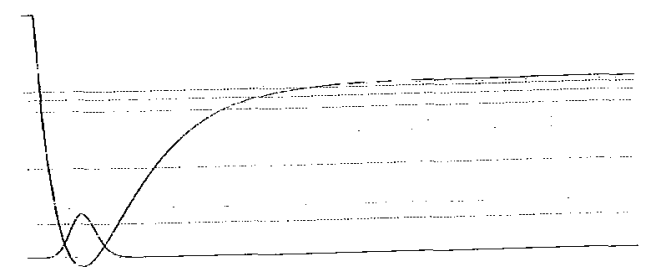

Fig. 5. The graph of the Morse potential with eigenenergies and the ground state wave function, obtained with the AMSSE program

\section{4. Konwent potential (double-well Morse potential)}

Because the simplest symmetric double-well potential - anharmonic oscillator of the $a x^{2}+b x^{4}$ type - is not solvable analytically, the Konwent potential [29-32], which has a simple form, has been used instead:

$$
V(x)=\frac{(\alpha \cdot \hbar)^{2}}{8 m_{e}} \cdot(2 S+1)^{2} \cdot\left(\frac{B}{2 S+1} \cosh (\alpha \cdot x)-1\right)^{2} .
$$

For some values of $S$ and $B$ there exist analytical solutions. In the test, value $B=0.04$ was used, and $S$ was equal to 1 ; then

$$
\begin{gathered}
E_{0}=\frac{(\alpha \cdot \hbar)^{2}}{2 m_{e}} \cdot\left(\frac{7}{4}+\left(\frac{B}{2}\right)^{2}-\sqrt{\frac{1}{4}+B^{2}}\right), \\
E_{1}=\frac{(\alpha \cdot \hbar)^{2}}{2 m_{e}} \cdot\left(\frac{5}{4}+\left(\frac{B}{2}\right)^{2}\right), \\
E_{2}=\frac{(\alpha \cdot \hbar)^{2}}{2 m_{e}} \cdot\left(\frac{7}{4}+\left(\frac{B}{2}\right)^{2}+\sqrt{\frac{1}{4}+B^{2}}\right) .
\end{gathered}
$$

The $V(x)$ graph is shown in Fig. 6. 


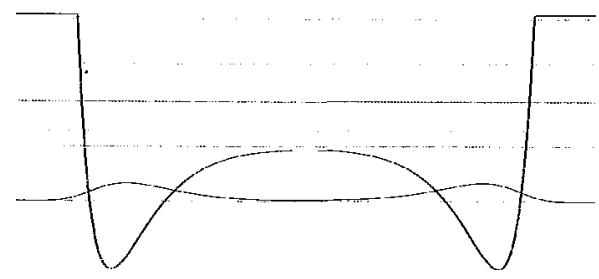

Fig. 6. The graph of the Konwent potential with eigenenergies and the ground state wave function, obtained with the AMSSE program

\section{5. Sources of errors in numerical algorithms}

Opposite to the approximate analytical methods of solving the Schrödinger equation, numerical methods are universal and, if properly applied, can quickly give accurate results. Analytical solutions often require great experience and intuition; incorrect assumptions can lead to significant errors. Below we discuss the influence of: (1) number of grid points $N$, (2) selected method of numerical approximation of the second derivative, (3) width $l=|b-a|$ of the integration range, on the quality and reliability of numerical results. As the measure of accuracy we take the relative error

$$
\delta E=\frac{\Delta E}{E_{\text {exact }}} \approx \frac{\Delta E}{E_{\text {numerical }}}=\frac{\left|E_{\text {exact }}-E_{\text {numerical }}\right|}{E_{\text {numerical }}}
$$

In Figures 8 and 9, we present dependences of accuracy defined above $(-\log \delta E$, right scales) and efficiency EF defined by (a) (left scales) on the number $N$ of grid points, for different algorithms. We point out, that Figs. 8 and 9 correspond to a harmonic oscillator potential and to the Coulomb potential $(l=2)$, respectively.

As we can see, both accuracy and efficiency increase with the increasing $N$, reach their maxima for some $N=N_{\text {max }}$ and then drop down.

For several methods, we estimated maximum accuracy, which can be theoretically achieved for given value of $N$. Our estimation is shown in Fig. 7. Let us note the linear dependence of numerical error on $N$. One can see that there is no sense in infinite increasing of $N$.

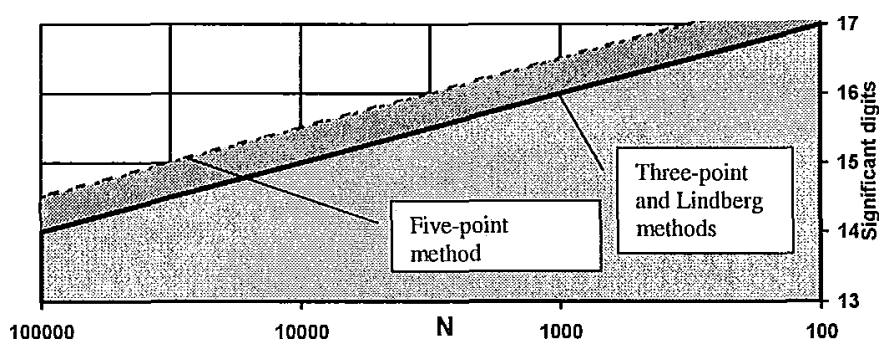

Fig. 7. Estimated possible magnitudes of numerical accuracy for three-point and five-point methods

The Guardiola-Ros algorithm needs separate analysis (Figs. 10 and 11), because it involves operations on dense matrices instead of band ones. Therefore, the quadratic dependence of numerical error on $N$ should be observed. 


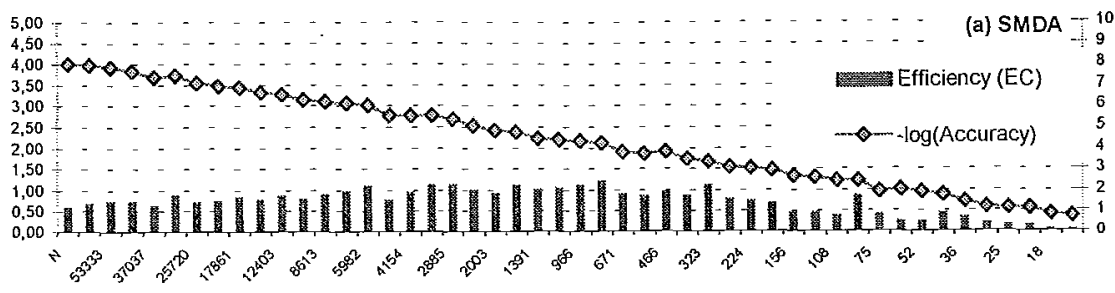

$N$
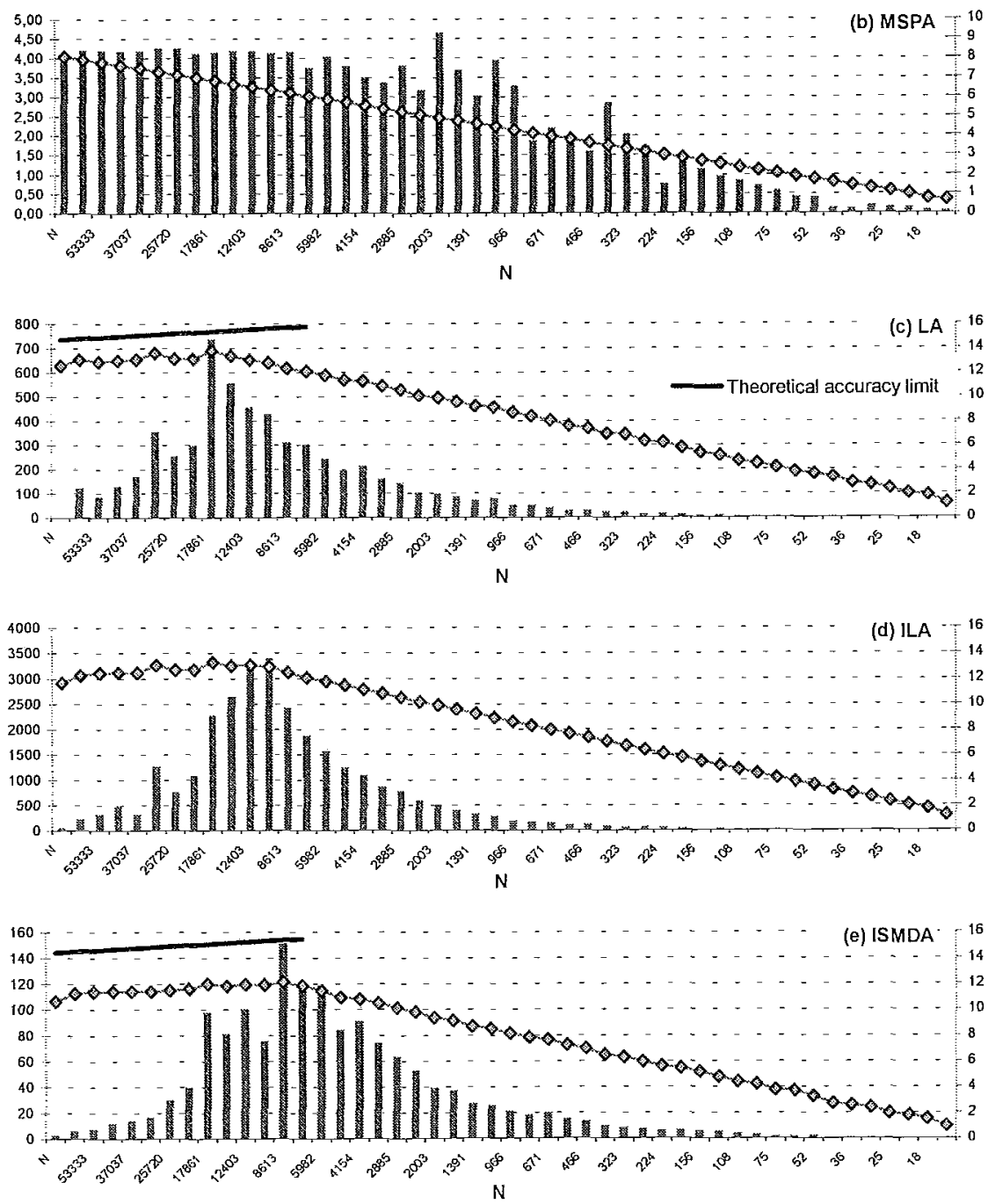

Fig. 8. Accuracy and efficiency as a function of the number of grid points $N$ for computational methods in case of harmonic oscillator potential 


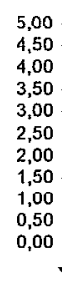

(a) SMDA
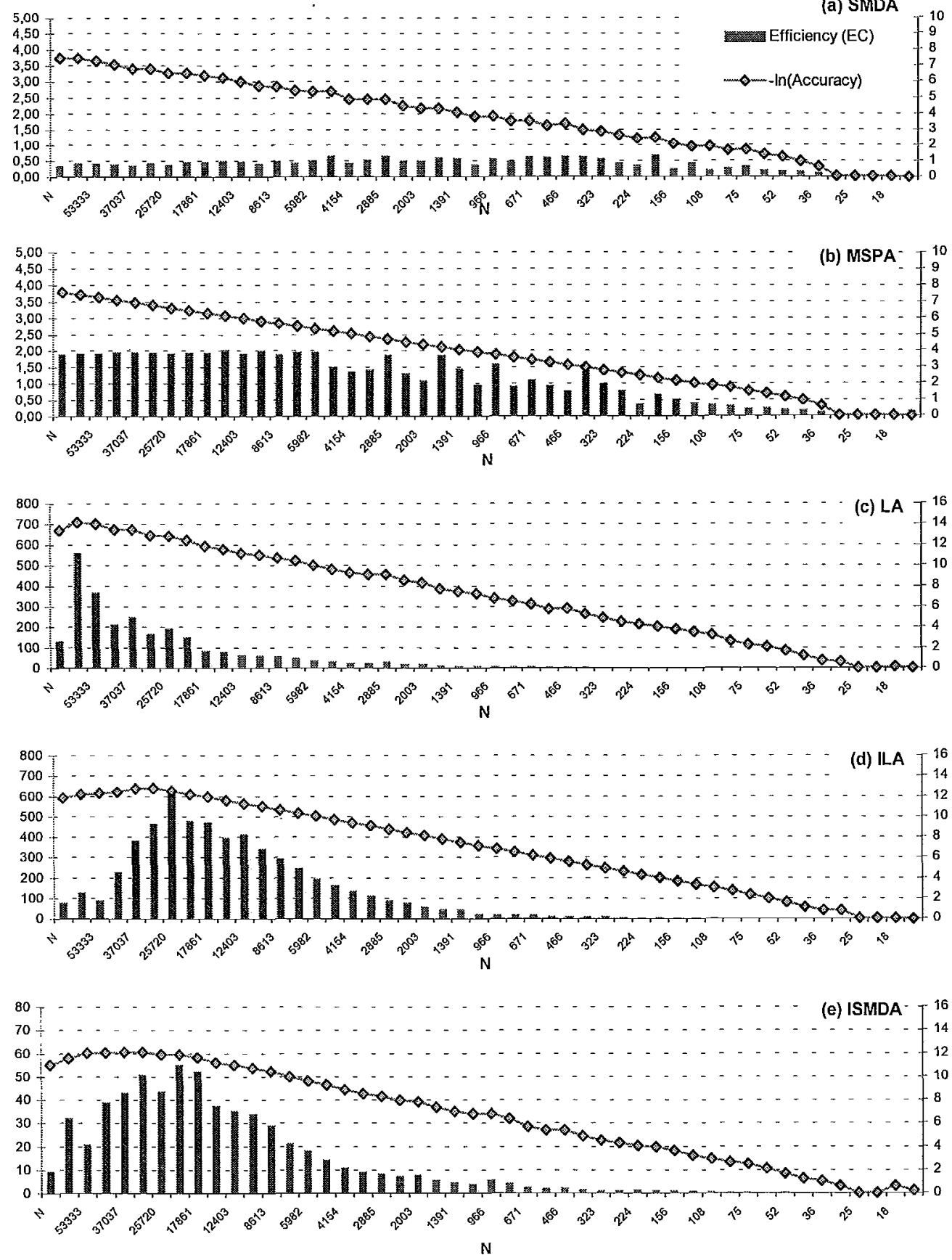

Fig. 9. Accuracy and efficiency as a function of the number of grid points $N$ for computational methods in case of Coulomb potential $(l=2)$ 


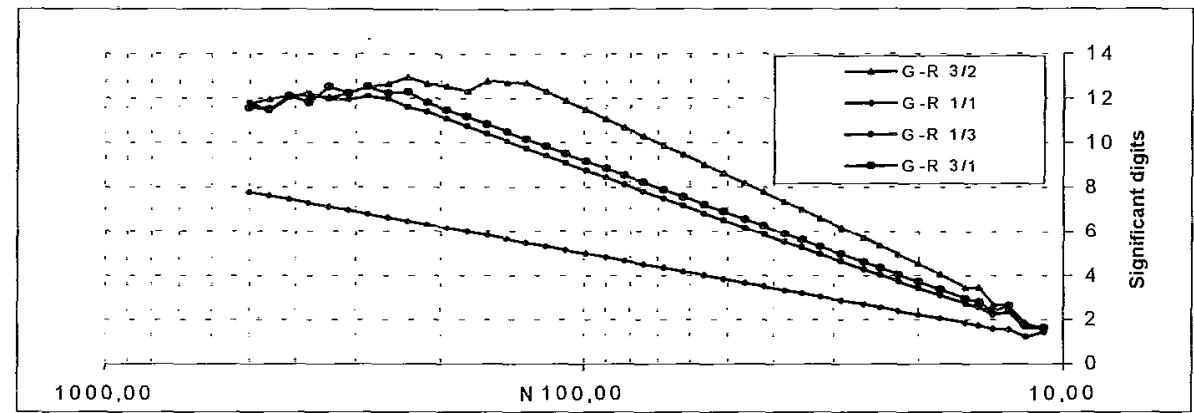

Fig. 10. Numerical results for Guardiola-Ros method with indicated Padé approximants of the second derivative operator for the harmonic oscillator potential

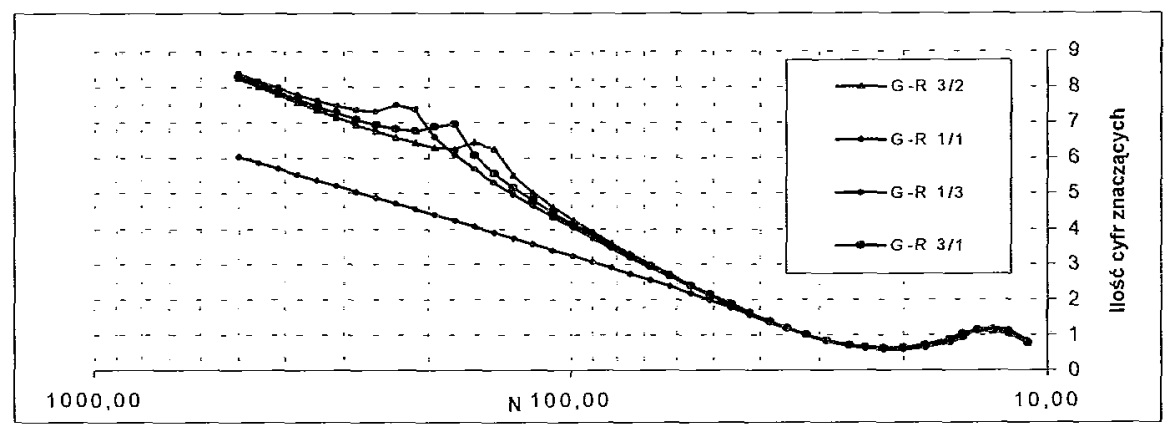

Fig. 11. Numerical results for the Guardiola-Ros method with indicated Padé approximants of the second derivative operator for the Coulomb potential $(l=1)$

\section{6. Influence of the second derivative approximation on the accuracy}

In the AMSSE program, we have applied four basic schemes of the second derivative approximation:

1. Three-point formula (3), accuracy $O\left(h^{2}\right)[22]$;

2. The Lindberg method (8), accuracy $O\left(h^{4}\right)$ [16];

3. Five-point formula (4), accuracy $O\left(h^{4}\right)$ [22];

4. The Guardiola-Ros method with [3/2] Padé approximant (7), accuracy $O\left(h^{10}\right)$ [15],

Grid number dependence of the achieved accuracy for methods 1-3 are depicted in Figs. 12 and 13

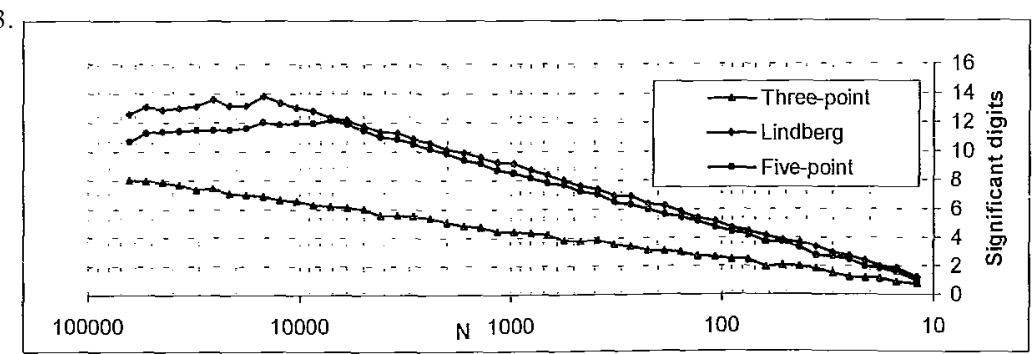

Fig. 12. Test results for harmonic oscillator potential 


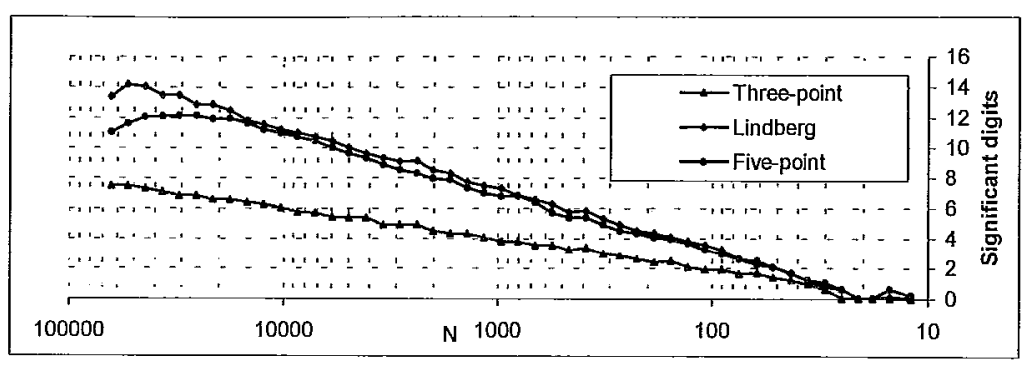

Fig. 13. Test results for Coulomb potential $(l=0)$

The Guardiola-Ros method needs separate discussion, because with the increasing $N$ the execution time rapidly grows. Despite, G-R method is specially precise for small $N$ and smooth potentials; for singular potentials the value of $N$, needed to obtain required accuracy, increases rapidly.

On the above graphs, the linear dependence of the number of significant digits on the logarithm of $N$ can be clearly seen, up to the maximum accuracy of given method. Because the number of significant digits is equal to $-\log \delta E$, the dependence of $\delta E$ on $N$ is of power type and the relation

$$
-\log (\delta E)=\mathrm{a} \cdot \log (N)+b
$$

is fulfilled at linear segments of each curve; in otherwords, $\delta E=10^{-b} \cdot N^{a}$.

Values of $a$ and $b$ depend on the selection of the second derivative approximation and on the potential energy function. The comparison results, collected in Table II, are interesting. For all tested potentials, the accuracy of three-point, Lindberg, and five-point methods is described by the formula

$$
\delta E=C^{\cdot} N^{-m},
$$

where $C$ is a potential-dependent constant. We point out that $a \approx m$ (cf. Table II), what means that the obtained numerical results are consistent with the order of second derivative approximation (its accuracy is $O\left(h^{m}\right)$ ).

Table II. Linear approximation coefficients for the accuracy of numerical algorithms

\begin{tabular}{ccccc}
\hline Potential & Method & $m$ & $a$ & $B$ \\
\hline \multirow{2}{*}{ Harmonic } & Three-point & 2 & 1.96 & -1.388 \\
oscillator & Five-point & 4 & 3.93 & -3.201 \\
& Lindberg & 4 & 4.00 & -2.991 \\
\hline \multirow{2}{*}{ Coulomb } & Three-point & 2 & 2.00 & -2.054 \\
$(l=0)$ & Five-point & 4 & 3.88 & -4.677 \\
& Lindberg & 4 & 4.00 & -4.764 \\
\hline \multirow{3}{*}{ Harmonic } & GRA [3/2] & 10 & 10.0 & -8.471 \\
oscillator & GRA [1/1] & 4 & 3.96 & -2.907 \\
& GRA [1/3] & 8 & 7.64 & -6.514 \\
& GRA [3/1] & 8 & 7.85 & -6.503 \\
\hline \multirow{3}{*}{ Coulomb } & GRA [3/2] & 10 & 6.3 & -8.232 \\
$(l=1)$ & GRA [1/1] & 4 & 4.07 & -4.943 \\
& GRA [1/3] & 8 & 6.8 & -9.334 \\
& GRA [3/1] & 8 & 6.5 & -8.768 \\
\hline
\end{tabular}




\section{7. Influence of the integration interval on the numerical results}

To take advantage of the implemented numerical algorithms, one has to be conscious of the assumptions made. The most important ones are the boundary conditions, which imply that the wave function $\Psi(a)=\Psi(b)=0$ and $\Psi(x)=0$ outside the integration interval. From the physical point of view it means that we put infinite potential barriers at $x=a$ and $x=b$.

The problem is particularly important for finite-value potentials defined on infinite intervals. The integration interval should be then chosen carefully in such a way, that the influence of artificial infinite barriers could be neglected. One cannot simply extend the integration interval in an uncontrolled way, because of the side effect of such an action (increasing $N$ or $h$ above their optimal values) which would decrease accuracy and/or make the computations significantly slower.

Let us note that in the case of singular potentials (e. g., Coulomb potential with $l=0$ ), the imposed boundary conditions result in a limited number of eigenvalues (the number of eigenvalues should be infinite); the highest eigenvalues are computed with lower precision.

The tests have shown, that in most cases the problem of integration interval selection can be solved. As an example, in Fig. 14 we present the results obtained for the Morse potential with improperly and properly chosen values of $a$ and $b$.

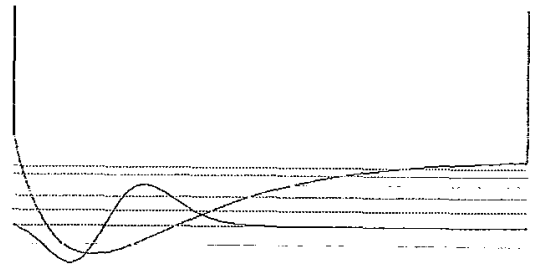

(a)

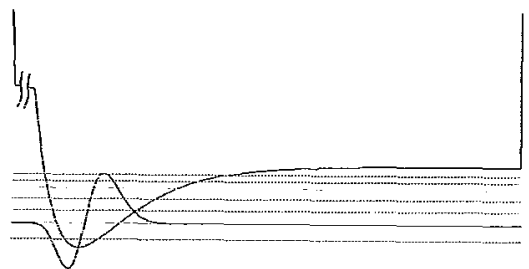

(b)

Fig. 14. (a) Calculated eigenenergies $\left(E_{0}=1.0013 \mathrm{E}-2 ; E_{1}=2.8486 \mathrm{E}-2 ; E_{2}=4.4904 \mathrm{E}-2\right)$. The accuracy is 3.7 E-3 (3 significant digits); the integration interval has not been chosen properly, (b) Calculated eigenenergies $\left(E_{0}=9.999999999999 \mathrm{E}-2 ; \quad E_{1}=2.841995766068 \mathrm{E}-2 ; \quad E_{2}=4.473319220241 \mathrm{E}-2\right)$. The accuracy is $1.4 \mathrm{E}-13$ (13 significant digits); the integration interval has been chosen properly. The wave function of the second eigenstate is also shown

A particle mass was chosen in such that the lowest eigenenergy takes the value 0.1. Parameter $a$ (left integration limit) influences calculated eigenenergy and eigenfunction accuracy. A behaviour of the computed eigenfunction near the boundaries is a signature of the proper choice of the integration limits. If the potential energy values are finite the eigenfunction tends to zero asymptotically. Such behaviour is shown in Fig. 15a, and corresponds to a correct choice of the integration range. The boundary condition $V(a)=\infty$ can substantially modify the behaviour of the computed wave function near the point $x=a$, when $a$ is chosen incorrectly. This case is depicted in Fig. 15b. 


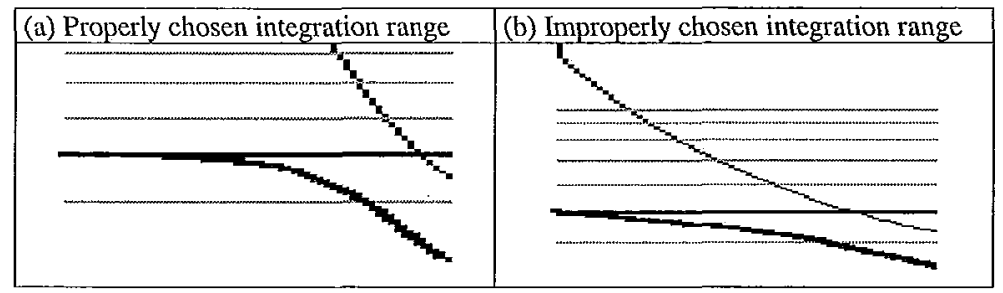

Fig. 15. Behaviour of the eigenfunction near the left integration limit in the discussed cases

\section{8. Other important results of the tests}

Many test results have already been presented along with the discussion of accuracy. However, for the practical point of view, the most important are the results obtained with the help of the testing worksheet mentioned at the beginning of Section 6 . The results are available to the user in two forms:

- Graphical - as a three-dimensional graph showing the time needed by a given method to achieve the required accuracy of determination of a single eigenvalue,

- Tabular - as a collection of call parameters and corresponding accuracy.

The obtained graphs and tables are shown in the Appendix in Figs. A1-A5. For each method and each required accuracy, there are shown: number $N$ of grid points needed (upper sub-row) and the accuracy actually achieved (lower sub-row).

The input data for all algorithms and for all test potentials were the same. The integration limits were properly chosen to eliminate errors (cf., Sec. 6.7). The testing process was automated. This way we obtained comprehensive results, which allow:

1. Comparison of the accuracy and efficiency of the implemented algorithms for the basic classes of potentials,

2. Selection of the most accurate, fastest and stable method and the optimal value of $N$ for a given practical problem (by fitting it into one of the classes) and, therefore, shortening the time needed for its solution.

\section{EXAMPLES OF AMSSE PROGRAM USAGE}

The AMSSE program user is able to:

- perform complete and accurate calculations for any single or multiple potential well, given by a formula or drawn with mouse, and to interpret experimental results or verify analytical solutions,

- analyse an influence of potential profile on the energy spectrum and wave functions by immediate comparison of the results before and after the change,

- perform series of calculations for a varying parameter in the formula describing potential energy, and to study its influence.

Below we present three examples of results obtained with the AMSSE program. 


\subsection{Quantum anharmonic oscillator}

The double potential well is described by the formula $V(x)=a x^{4}-b x^{2}+c x$. If $c=0$, the potential is symmetric and the lowest eigenergies can be quasi-degenerated (their values can differ less than machine epsilon), cf., Fig. 16a. Increasing of $c$ (what corresponds to nonsymmetric double well) leads to removal of the double quasi-degeneracy (Figs. 16b-d).

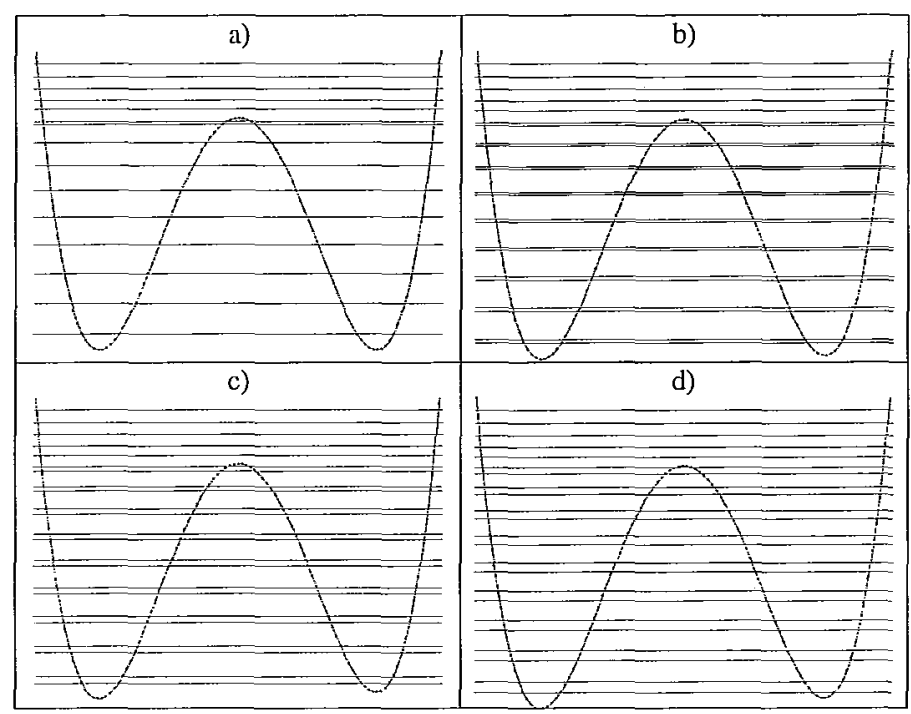

Fig. 16. Graphs of the anharmonic oscillator potential with calculated eigenergies

\section{2. Single and double finite rectangular quantum well}

With the AMSSE program, we have calculated eigenergies and eigenfunctions of charge carriers with effective-mass of $0.07 m_{e}$ in the rectangular potential well $0.75 \mathrm{eV}$-deep and $10 \mathrm{~nm}$-wide (cf., Fig. 17a), and in the same well with a $2.5 \mathrm{~nm}$-thick barrier in the middle (cf., Fig. 17b). Let us note, that in the double-well case the eigenstates appear in quasi-degenerated-pairs.

Parameters used in the example correspond to GaAs/AlGaAs semiconductor heterostructures.
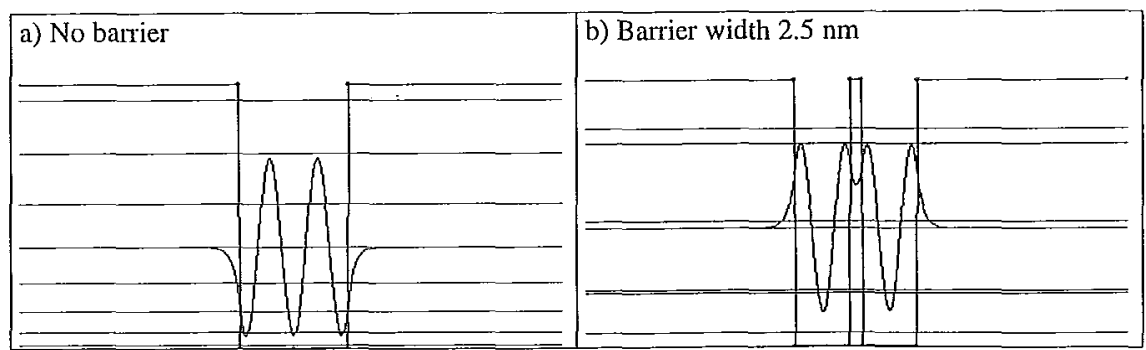

Fig. 17. Single and double rectangular quantum well 


\section{3. Band structure of one-dimensional solid}

We have calculated eigenvalues and eigenfunctions of electrons moving in a periodic potential given by

$$
V(x)=V_{0} \cdot|\sin a x| .
$$

The result are presented in Figs. 18 and 19.

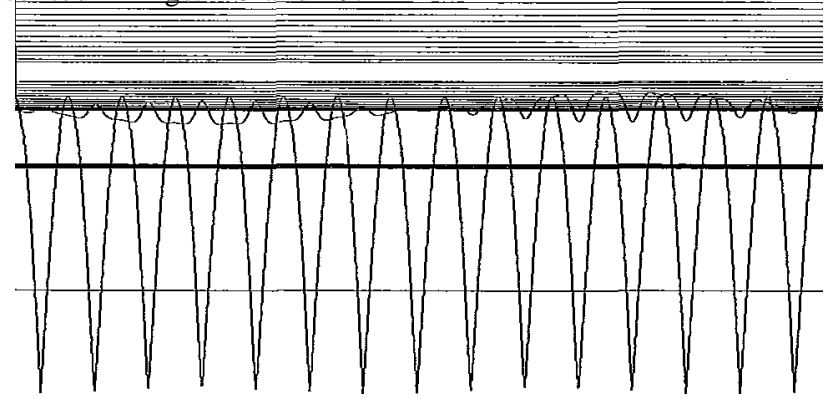

Fig. 18. Band structure and an example wave function in one-dimensional crystal

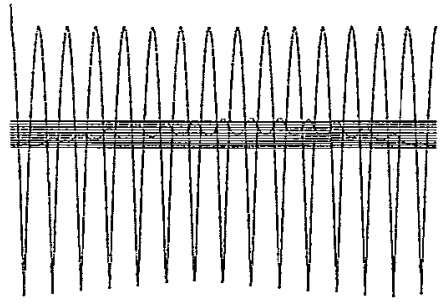

(a)

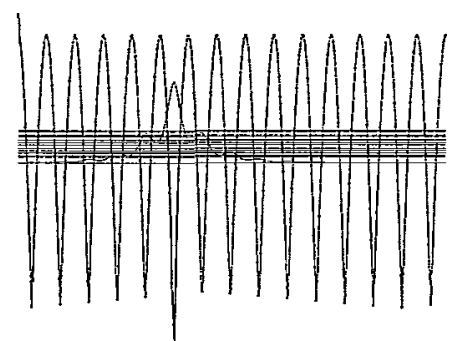

(b)

Fig. 19. The electronic wave function in a perfect lattice (a) and in lattice with an impurity or point defect, which causes electron localization (b)

\section{MATRIX REPRESENTATION OF THE EFFECTIVE-MASS EQUATION}

Physical properties of low-dimensional semiconductor heterostructures (e. g., quantum wells and superlattices) are commonly interpreted theoretically within the effective-mass theory [11], In this context the main task is aimed towards an accurate solution of onedimensional effective-mass equation for envelope function

$$
-\frac{\hbar^{2}}{2} \frac{\mathrm{d}}{\mathrm{d} x} \frac{1}{m(x)} \frac{\mathrm{d} \Psi(x)}{\mathrm{d} x}+V(x) \Psi(x)=\varepsilon \Psi(x)
$$

with the charge carrier mass $m$ being a function of $x$ coordinate (the $O X$ axis is assumed parallel to the growth direction of the heterostructure).

In order to discretize the above equation, we introduce the second grid of points, shifted by $h / 2$ relative to the main grid: 


$$
x_{i+1 / 2}=a+\left(i+\frac{1}{2}\right) h
$$

Applying three-point formula for the first derivative twice, we obtain for each $x_{i}$

$$
-\left[\frac{1}{m_{i-1 / 2}} \Psi_{i-1}-\left(\frac{1}{m_{i-1 / 2}}+\frac{1}{m_{i+1 / 2}}\right) \Psi_{i}+\frac{1}{m_{i+1 / 2}} \Psi_{i+1}\right]+\frac{2 h^{2}}{\hbar^{2}}\left(V_{i}-\varepsilon\right) \Psi_{i}=0
$$

Therefore, the effective-mass equation is reduced to an eigenproblem for a symmetic tridiagonal matrix $M_{r}=\operatorname{trid}\left(1 / m_{i-1 / 2}, 1 / m_{i-1 / 2}+1 / m_{i+1 / 2}+2 h^{2} V / h^{2},-1 / m_{i+1 / 2}\right.$. This discrete form of (11) satisfies continuity conditions for the envelope function $\Psi$ and $(1 / m) d \Psi / d x$ in the limit of $h \rightarrow 0$ [11].

Eigenvectors of $M_{T}$ can be found with DWSZ method (9a-f). Some results of accuracy test for the discrete effective-mass equation applied to several semiconductor hetero-structures have been published [11]. A simple way of taking into account the conduction band nonparabolicity effects is also presented there.

\section{FINAL CONCLUSIONS}

The AMSSE program we have created and described in this paper is an efficient numerical tool for solving stationary one-dimensional Schrodinger equation. Its efficiency and high accuracy result from the use of algebraic matrix approach, which allows us to apply advanced algorithms worked out in numerical linear algebra.

The main capabilities of the AMSSE program can be summarized as follows:

1. It is a stable, fast and efficient computational platform for solving stationary onedimensional Schrodinger equation with arbitrary analytical or numerical potentials.

2. It allows to calculate and to display in its graphical interface a large number of eigenvalues and eigenvectors at the same time.

3. It has a lot of different efficient algorithms built-in, which allow us to compare results obtained with different methods.

4. It allows the user to select the numerical algorithm most suitable for considered practical problem. It allows us also to choose an optimum number of grid points for the required accuracy.

5. It can be applied for scientific purposes in the field of quantum mechanics and lowdimensional physics.

6. It fully uses capabilities and functions of Windows operating system, which makes it a user-friendly and comfortable tool.

7. It is a valuable tool for physicists, chemists, engineers and nanotechnologists.

In order to obtain the AMSSE program, please contact its authors. 


\section{Appendix - comprehensive test results}

For each method and for each required accuracy, number $N$ of grid points needed (upper sub-row) and the accuracy actually achieved (lower sub-row) are shown. Detailed description can be found in Section 6 .

\begin{tabular}{|c|c|c|c|c|c|c|c|c|c|c|c|c|c|c|}
\hline & Ete & 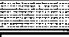 & 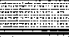 & H= & H= & $\mathrm{Reg}$ & IIPO & accu & acy & Ant & w & El & $=$ & $=$ \\
\hline Method $d=t$ & BidE=01: & EOGE & T10E: & $10 E=4$ & $10 E=0$ & $10 E=\underline{0 E}$ & $11,0 \mathrm{E}=0$ & 1OEE & $10 \mathrm{E}=0$ & 10 & $100=11$ & Wíne & TOEE & $=-m$ \\
\hline MSPA: & 20 & 60 & 215 & 642 & 1916 & 6866 & 20501 & & & & & & & \\
\hline 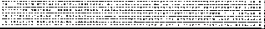 & $7.9 E-02$ & $9,0 \mathrm{E}-03$ & $7,1 \mathrm{E}-04$ & $8,1 \mathrm{E}-05$ & $9,1 E-06$ & $7,1 \mathrm{E}-07$ & $7,9 E-08$ & & & & & & & \\
\hline $5 \mathrm{MDA}=$ & 20 & 60 & 215 & 642 & 1916 & 6866 & 20501 & & & & & & & \\
\hline 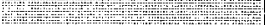 & $6,9 E-02$ & $9,1 \mathrm{E}-03$ & $8,3 E-04$ & 8,5E-05 & $3,2 E-06$ & $7,7 E-07$ & $8,8 \mathrm{E}-08$ & & & & & & & \\
\hline 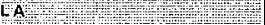 & 12 & 20 & 35 & 60 & 103 & 179 & 372 & 642 & 1109 & 1916 & 3311 & 5722 & 11864 & \\
\hline 10 & $6.3 E-02$ & $6.5 E-03$ & $6,9 E-04$ & $8,5 E-05$ & $8,2 E-06$ & $9,5 E-07$ & $6,1 E-08$ & $5.9 \mathrm{E}-09$ & $7.1 \mathrm{E}-10$ & $7.5 \mathrm{E}-11$ & $8,5 E-12$ & $9,5 E-13$ & $3,5 E-14$ & \\
\hline MLAT & 12 & 20 & 35 & 60 & 124 & 215 & 372 & 642 & 1109 & 1916 & 4768 & & & \\
\hline 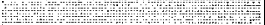 & $6,1 E-02$ & $5,6 E-03$ & $6,4 E-04$ & $7,7 \mathrm{E}-05$ & $5,8 E-06$ & $6,9 E-07$ & $5,4 E-08$ & $6,1 E-09$ & $6,9 E-10$ & $8,3 E-11$ & $8,4 E-12$ & & & \\
\hline $\mathrm{MHA}+\mathrm{P}$ & 12 & 20 & 35 & 60 & 103 & 179 & 372 & 642 & 1109 & 1916 & 3311 & 5722 & 11864 & \\
\hline 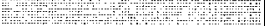 & 6,1E-02 & $5,8 \mathrm{E}-03$ & $6.4 \mathrm{E} \cdot 04$ & $7,6 \mathrm{E}-05$ & $8,9 \mathrm{E}-06$ & $9,9 E-07$ & $5,4 E-08$ & $6,1 E-09$ & $6,8 E-10$ & $7.7 \mathrm{E}-11$ & $8,6 E-12$ & $9,8 E-13$ & $3,7 E-14$ & \\
\hline & 12 & 20 & 35 & 60 & 103 & 179 & 372 & 642 & 1109 & 1916 & 3311 & 5722 & & \\
\hline 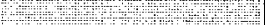 & $6,1 E-02$ & $5,8 E-03$ & $6,4 E-04$ & $7,6 E-05$ & $8,9 E-06$ & $9,9 E-07 \mid$ & $5,4 E-08$ & $6,1 \mathrm{E}-09$ & $6,8 E-10$ & $7,7 \mathrm{E}-11$ & $8,6 E-12$ & $9,7 E-13$ & & \\
\hline 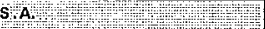 & 20 & 60 & 215 & 642 & 1916 & 6866 & 6866 & & & & & & & \\
\hline 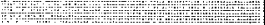 & $7,9 \mathrm{E}-02$ & $9,0 E-03$ & $7,1 E-04$ & $8,1 E-05$ & $9,1 E-06$ & 7,1E-07 & $7,1 E-07$ & & & & & & & \\
\hline $\mathrm{R} P$ & 20 & 60 & 215 & 770 & & & & & & & & & & \\
\hline 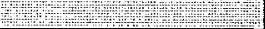 & 7,9E-02 & $9,0 E \cdot 03$ & $7,6 \mathrm{E}-04$ & $3,6 \mathrm{E}-05$ & & & & & & & & & & \\
\hline ISMOA $\quad$ A & 14 & 24 & 42 & 72 & 149 & 258 & 446 & 770 & 1331 & 2299 & 4768 & 6866 & & \\
\hline 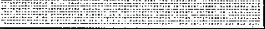 & $4,0 E-02$ & $6,5 E-03$ & $6,9 E \cdot 04$ & $9,6 \mathrm{E}-05$ & 5,7E-06 & $6,3 \mathrm{E}-07$ & $7,2 E-08$ & $8,3 E-09$ & $8,8 \mathrm{E}-10$ & $9,7 \mathrm{E}-11$ & $7,1 E-12$ & $7,05-13$ & & \\
\hline $\mathrm{GRA}, \mathrm{U}$ & 10 & 14 & 17 & 20 & 24 & 29 & 42 & 50 & 60 & 72 & 103 & 124 & & \\
\hline $4+4=4+4=$ & $8,0 E-02$ & $2,0 E-03$ & $1,5 E-04$ & $3,0 E-05$ & 4,7E-06 & $6,8 \mathrm{E}-07$ & $1,7 E-08$ & $2,9 \mathrm{E}-09$ & $4,7 E-10$ & $7,7 \mathrm{E}-11$ & $2.1 \mathrm{E}-12$ & $4,2 \mathrm{E}-13$ & & \\
\hline 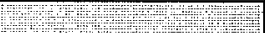 & & & & & & & & & & & & & & \\
\hline 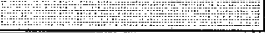 & & & & & & & & & & & & & & \\
\hline
\end{tabular}

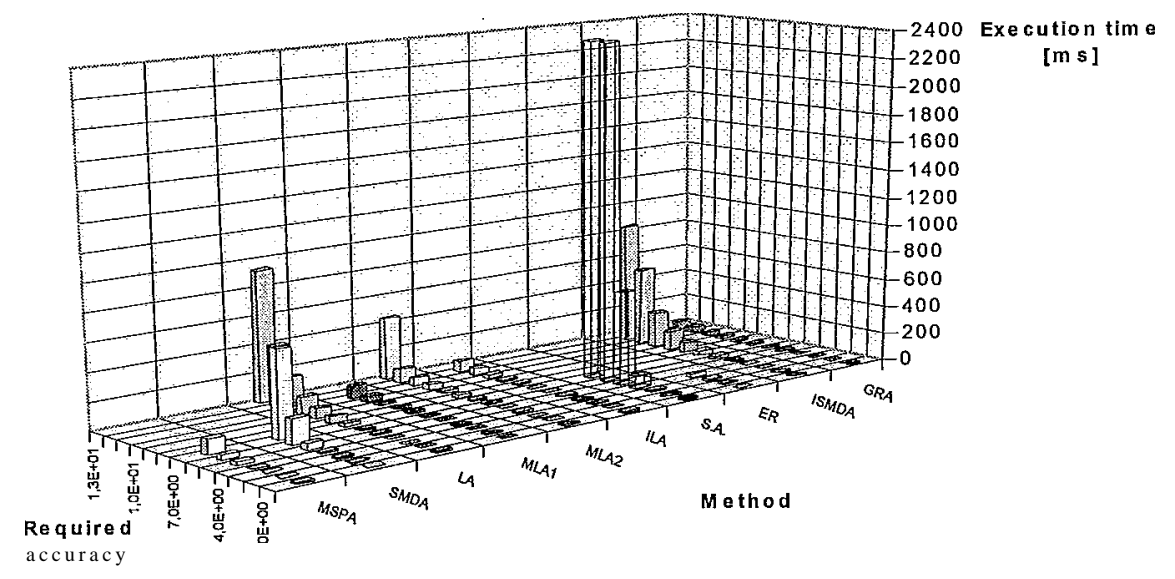

Fig. Al. Test results for the harmonic oscillator potential 


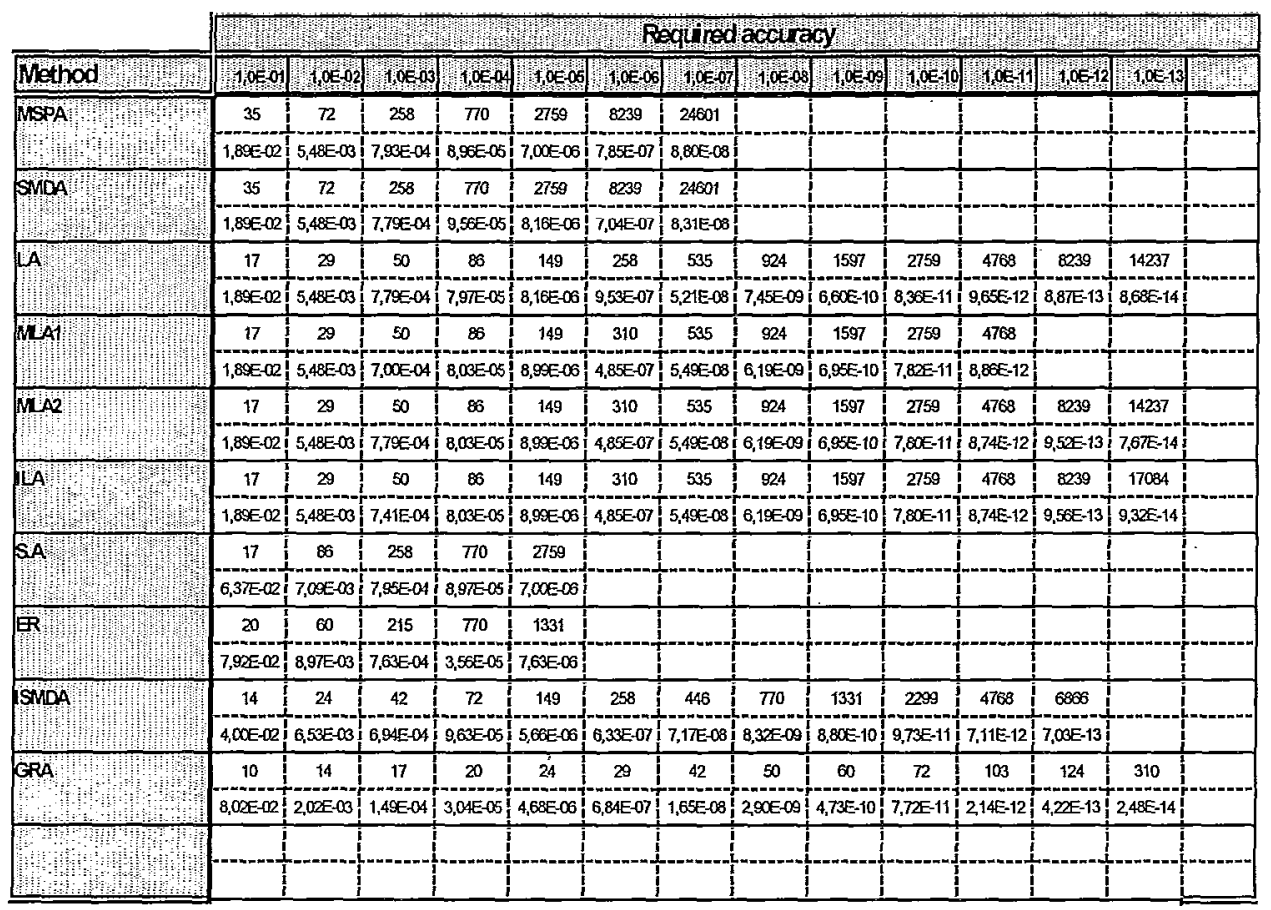

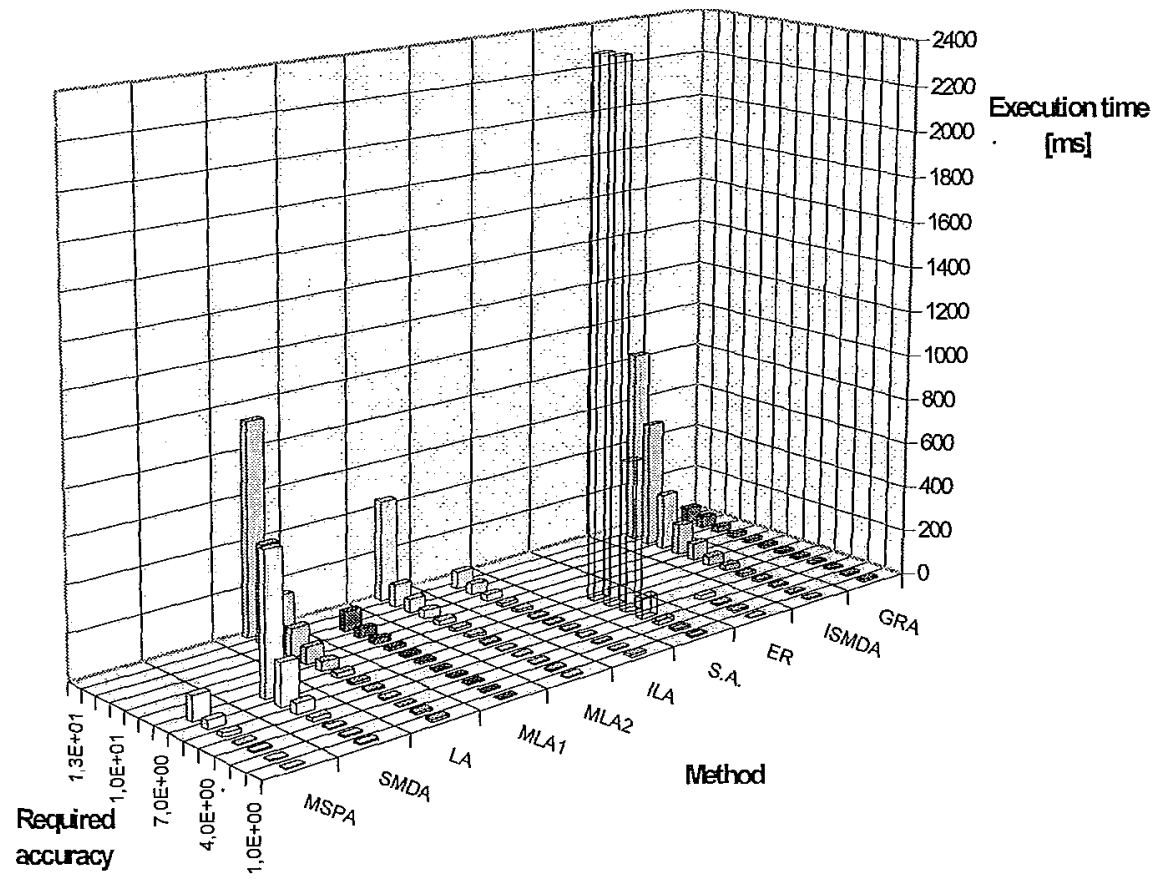

Fig. A4. Test results for the Coulomb potential 


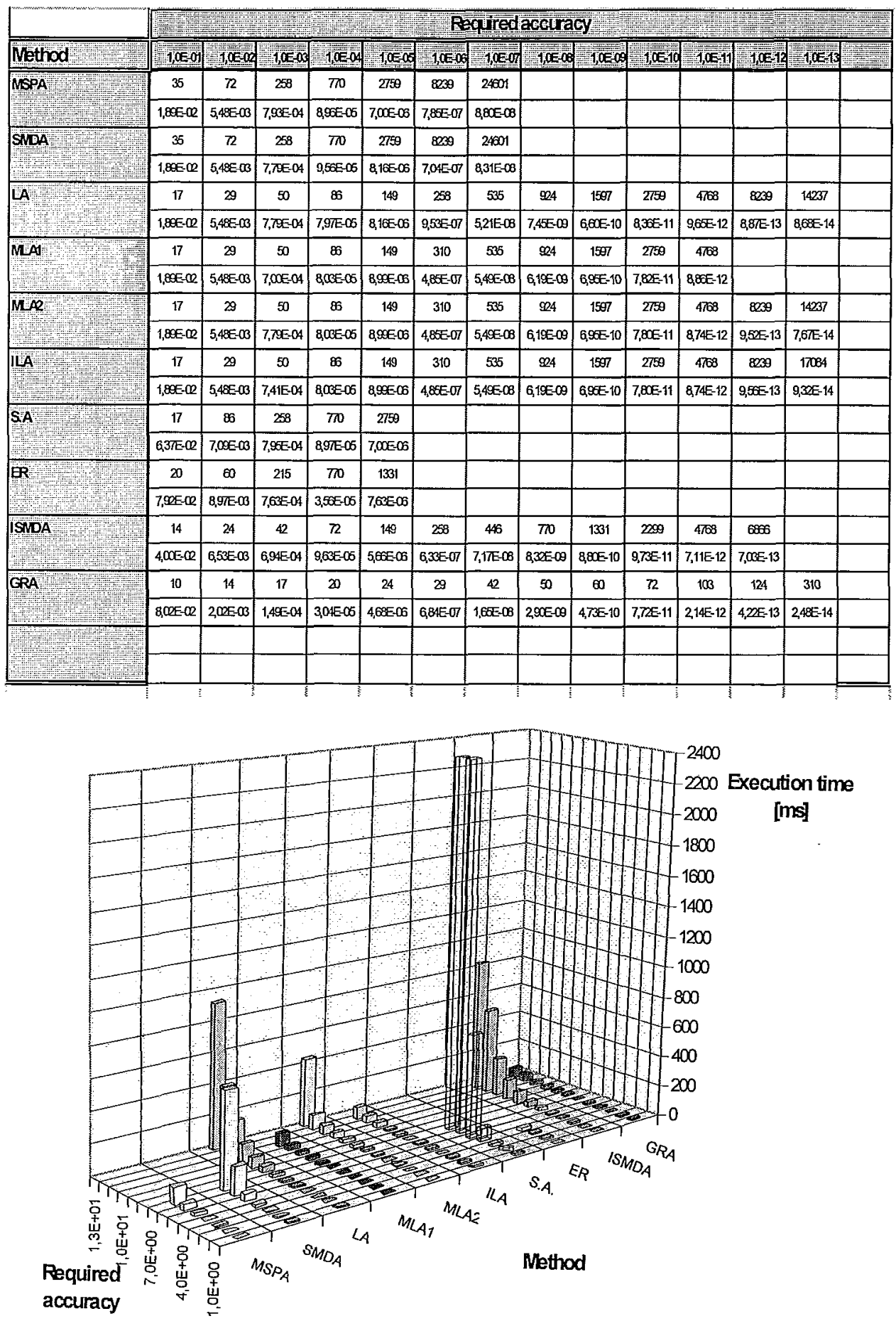

Fig. A3. Test results for the Konwent potential 


\begin{tabular}{|c|c|c|c|c|c|c|c|c|c|c|c|c|c|c|}
\hline \multirow[b]{2}{*}{ Method } & \multicolumn{14}{|c|}{ Tot $\quad$ Required accuracy } \\
\hline & $10 E-1$ & $100-02$ & $1,0=03$ & DOE:04 & $1,0=05$ & $1,05.06$ & $1: 00-07$ & $100=0$ & $11,00=09$ & $1,0 \mathrm{E}+10$ & $1,06+11$ & $100=12$ & 1,0 E 33 & \\
\hline \multirow[t]{2}{*}{ MSPA } & 179 & 535 & 1916 & 5722 & 17084 & & & & & & & & & \\
\hline & $7,6 \mathrm{E}-02$ & $9,4 \mathrm{E}-03$ & $7,5 E-04$ & $18,4 E-05$ & $9,4 \mathrm{E}-06$ & & & & & & & & & \\
\hline \multirow[t]{2}{*}{ SMDA } & 179 & 535 & 1916 & 5722 & 17084 & & & & & & & & & \\
\hline & $7,6 \mathrm{E}-02$ & $8,2 \mathrm{E}-03$ & $7,0 E-04$ & $8.1 \mathrm{E}-05$ & $9,3 \mathrm{E}-06$ & & & & & & & & & \\
\hline \multirow{2}{*}{ A } & 258 & 924 & 3311 & 9887 & 35425 & & & & & & & & & \\
\hline & $9,0 E-02$ & $9,9 E-03$ & $8,1 E-04$ & $8,1 E-05$ & $7,7 E-06$ & & & & & & & & & \\
\hline \multirow{2}{*}{ MoAt } & 258 & 924 & 2759 & 9887 & 29521 & & & & & & & & & \\
\hline & $8,6 \mathrm{E}-02$ & $8,7 \mathrm{E}-03$ & $9,2 \mathrm{E}-04$ & $8,9 E-05$ & $9,6 E-06$ & & & & & & & & & \\
\hline \multirow{2}{*}{ MLA2 } & 258 & 924 & 3311 & 9887 & 35425 & & & & & & & & & \\
\hline & $9,1 E-02$ & $9,2 \mathrm{E}-03$ & $7,8 \mathrm{E}-04$ & $8,9 E-05$ & 7.0E-06 & & & & & & & & & \\
\hline \multirow[t]{2}{*}{$\mathrm{A}$} & 258 & 924 & 3311 & 9887 & 35425 & & & & & & & & & \\
\hline & $9,0 \mathrm{E}-02$ & $9,2 E-03$ & $7,8 \mathrm{E}-04$ & $8,9 \mathrm{E}-05$ & $7,0 E-06$ & & & & & & & & & \\
\hline \multirow[t]{2}{*}{ S.A. } & 179 & 535 & 1916 & 5722 & 8239 & & & & & & & & & \\
\hline & $7,6 \mathrm{E}-021$ & $9,4 \mathrm{E}-03$ & $7,5 \mathrm{E}-04$ & $8,4 E-05$ & $4,0 E-05$ & & & & & & & & & \\
\hline \multirow[t]{2}{*}{ ER } & 179 & 535 & 1916 & & & & & & & & & & & \\
\hline & $7,7 \mathrm{E}-02$ & $9.3 \mathrm{E}-03$ & $7,1 E-04$ & & & & & & & & & & & \\
\hline \multirow{2}{*}{ SMPA } & 446 & 3973 & & & & & & & & & & & & \\
\hline & $9,0 \mathrm{E}-02$ & $9,9 \mathrm{E}-03$ & & & & & & & & & & & & \\
\hline \multirow{2}{*}{\begin{tabular}{|l|l|} 
GRA & \\
& \\
\end{tabular}} & 258 & 770 & & & & & & & & & & & & \\
\hline & $8,9 E-02$ & $1,3 \mathrm{E}-02$ & & & & & & & & & & & & \\
\hline 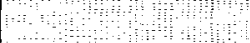 & & & & & & & & & & & & & & \\
\hline & & & & & & & & & & & & & & \\
\hline
\end{tabular}

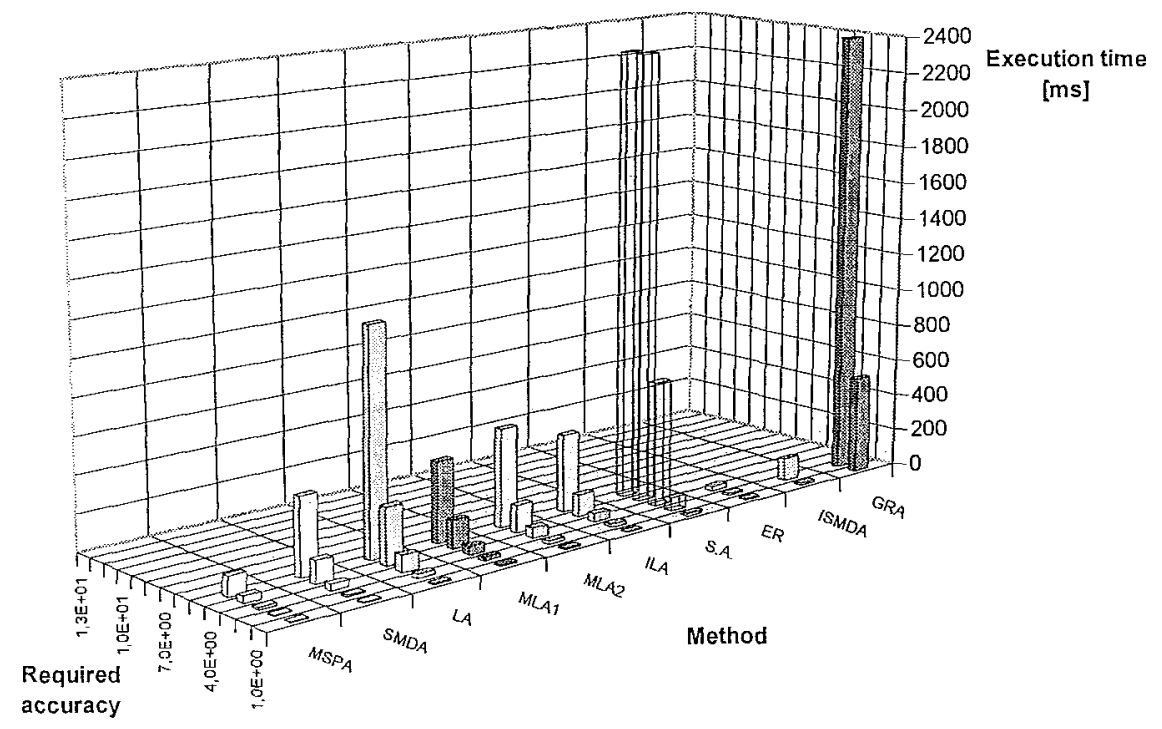

Fig. A4. Test results for the Coulomb potential $(l=0)$ 


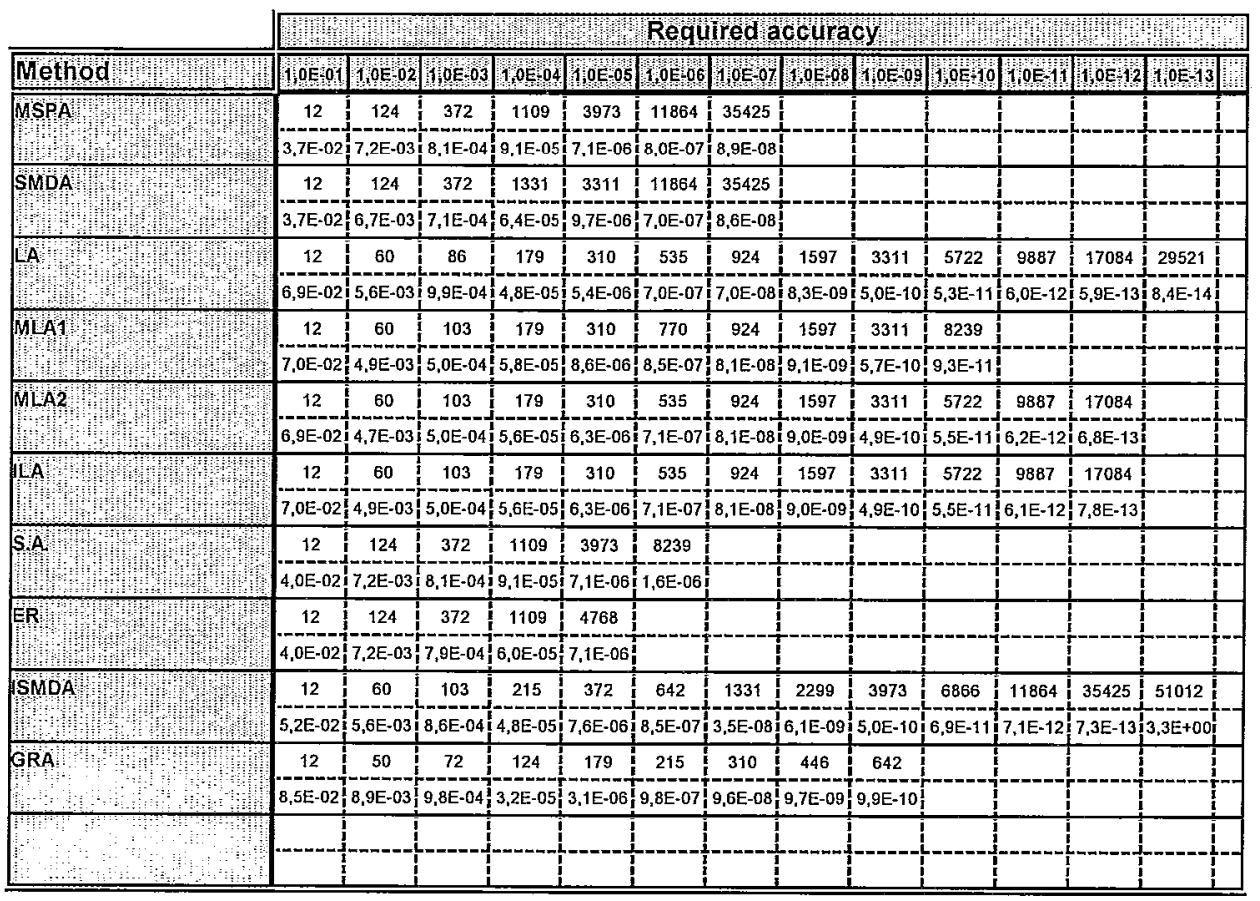

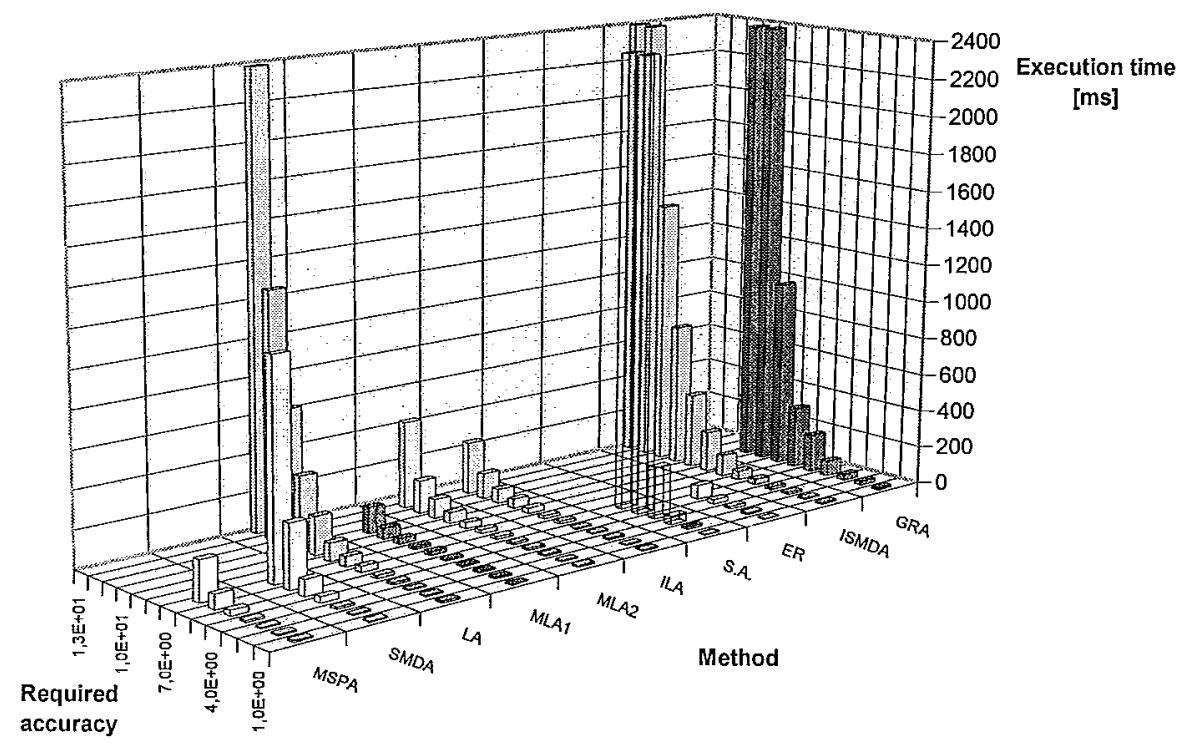

Fig. A5. Test results for the Coulomb potential $(l=2)$ 


\section{References}

[1] L. D. Landau, E. M. Lifshitz, Course of Theoretical Physics. Vol. Ill Quantum Mechanics, 4th ed,, Nauka, Moscow (1989) (in Russian).

[2] E. Merzbacher, Quantum Mechanics, 3rd ed., John Wiley \& Sons, New York (1998).

[3] R. L. Liboff, Introductoiy Quantum Mechanics, 3rd ed., Addison-Wesley Longman, New York, (1998).

[4] W. Greiner, Quantum Mechanics. An Introduction, 2nd corrected ed., Springer-Verlag, Berlin (1993).

[5] S. Flügge, Practical Quantum Mechanics, Springer-Verlag, Berlin (1999).

[6] R. Shankar, Principles of Quantum Mechanics, 2nd ed., Plenum Press, N.Y. and London (1994).

[7] F. Mand1, Quantum Mechanics, Wiley, Chichester (1992).

[8] J. J. Sakurai, Modern Quantum Mechanics, Addison-Wesley, Reading (1994).

[9] F. Schwabl, Quantum Mechanics, 2nd revised ed., Springer-Verlag, Berlin (1995).

[10] J. F. Van der Maelen Uria, S. Garcia-Granda, A. Menendez-Veläzquez, Am. J. Phys. 64, 327 (1996).

[11] W. Salejda, M. H. Tyc, J. Andrzejewski, M. Kubisa, J. Misiewicz, M. Just, K. Ryczko, Acta Phys. Pol., 95, 881 (1999).

[12] J. Killingbeck, Microcomputer Algorithms, Hilger, Bristol, 1991; J. Killingbeck and G. Jolicard, Phys. Lett. A172, 313 (1993).

[13] P. Harrison, Quantum Wells, Wires and Dots. Theoretical and Computational Physics, Wiley, Chichester, Ch. 2 and 3 (2000).

[14] W. H. Press, B. P. Flannery, S. A. Teukolsky, W. T. Vetterling, Numerical Recipes. Art of Scientific Computing. Cambridge University Press, Cambridge, Ch. 16 (1992).

[15] R. Guardiola, J. Ros, J. Comput. Phys. 45, 374 (1982).

[16] B. Lindberg, J. Chem. Phys. 88, 3805 (1988).

[17] G. C. Groenenboom and H. M. Buck, J. Chem. Phys. 92, 4374 (1990).

[18] Ch. 11 in [14],

[19] J. H. Wilkinson, The Algebraic Eigenvalue Problem, Clarendon Press, Oxford 1965; see also Handbookfor Automatic Computations, vol. 2, Linear Algebra, J. H. Wilkinson and C. Reinsch (Eds.), Springer-Verlag, Heidelberg (1971).

[20] E. Anderson et al. LAPACK Users' Guide, 3rd ed., SIAM, Philadelphia (2000).

[21] B. T. Smith et al, Matrix eigensystems routines - EISPACK guide, Lecture Notes in Computer Science, vol. 6, Berlin, Springer, Sec. Ed. (1976); B. W. Garbow, J. M. Boyle, J. J. Dongara, C. B. Moler, Matrix eigensystems routines - EISPACK guide extension, Lecture Notes in Computer Science, vol. 61, Berlin, Springer (1977).

[22] C. F. Gerald, P. O. Wheatley, Applied Numerical Analysis, Addison-Wesley, Reading (1989).

[23] M. Just, M. Sc. Thesis, Numerical Methods of Solving Schrödinger Equation. Program Package: MARKS, Report SPR-333/1998, Institute of Physics, Wroclaw University of Technology, Wroclaw 1998 (in Polish).

[24] A. Baker and P. Graves-Morris, Padé Approxitnants. Encyclopaedia of Mathematics and its Applications, vol. 13 and 14, Addison-Wesley (1981).

[25] P. Dean, Rev. Mod. Phys., 44, 127 (1972); see also W. Salejda, Int. J. Mod. Phys., B9, 1429 (1995); B9, 1453 (1995); B9, 1475 (1995).

[26] J. W. Demmel, Applied Numerical Linear Algebra, SIAM, Philadelphia (1996).

[27] K. S. Dy, S.Y. Wu, T. Spratlin, Phys. Rev. B20, 4237 (1979).

[28] Z. Zheng, J. Phys. C: Cond. Matt., 19, L689 (1986).

[29] H. Konwent, Phys. Lett. A118, 467 (1986).

[30] H. Konwent, Acta Phys. Pol. A71, 637 (1987).

[31] H. Konwent, Phys. Stat. Sol. B138, K7 (1986).

[32] H. Konwent, P. Machnikowski, P. Magnuszewski, A. Radosz, J. Phys. A: Math.Gen.. A31, 7541 (1998). 